\title{
Full observation of single-atom dynamics in cavity QED
}

\author{
H. Mabuchi, J. Ye, and H. J. Kimble \\ Norman Bridge Laboratory of Physics 12-33, California Institute of Technology, Pasadena, CA 91125 U.S.A.
}

(May 24, 1998)

\begin{abstract}
We report the use of broadband heterodyne spectroscopy to perform continuous measurement of the interaction energy $E_{\text {int }}$ between one atom and a high-finesse optical cavity, during individual transit events of $\sim 250 \mu$ s duration. We achieve a fractional sensitivity $\simeq 4 \times 10^{-4} / \sqrt{\mathrm{Hz}}$ to variations in $E_{\text {int }} / \hbar$ within a measurement bandwidth that covers 2.5 decades of frequency $(1-300 \mathrm{kHz})$. Our basic procedure is to drop cold Cesium atoms into the cavity from a magnetooptic trap while monitoring the cavity's complex optical susceptibility with a weak probe laser. The instantaneous value of the atom-cavity interaction energy, which in turn determines the coupled system's optical susceptibility, depends on both the atomic position and (Zeeman) internal state. Measurements over a wide range of atom-cavity detunings reveal the transition from resonant to dispersive coupling, via the transfer of atom-induced signals from the amplitude to the phase of light transmitted through the cavity. By suppressing all sources of excess technical noise, we approach a measurement regime in which the broadband photocurrent may be interpreted as a classical record of conditional quantum evolution in the sense of recently-developed quantum trajectory theories.
\end{abstract}

Optical cavity quantum electrodynamics (QED) in the strong coupling regime [1] provides a unique experimental paradigm for real-time observation of quantum dynamical processes at the single-atom level. While spectacular advances have certainly been made in the preparation and tomography of quantum states of motion for a single trapped ion [2]3], all such experiments have involved the accumulation of ensemble-averaged data over many successive realizations of the process being studied. Recent studies of single-molecule dynamics have likewise demonstrated the "immediate" detection of photochemical [t] or conformational [5] events, but such experiments presently lack the potential that cavity QED provides for observing quantum processes on a timescale that makes coherent control/intervention a tangible possibility. We wish here to look beyond the mere detection of quantum jumps, and to focus on the development of a broadband, singleshot measurement technique that achieves signal-to-noise ratio $>1$ over a bandwidth that includes all characterstic frequencies of a quantum dynamical process.

Real-time observation of quantum dynamics in manyatom systems has recently become an important theme in atomic physics, with notable demonstrations involving vibrational excitations of a trapped Bose-Einstein condensate [6] and the decay of coherent oscillations of an ensemble of atoms in an optical lattice [7,8]. In contrast to programs like these, for which the scientific emphasis lies on noninvasive observation of a system's intrinsic dynamical processes, experiments in single-atom cavity QED hold great potential for enabling precise investigations of how measurement backaction alters the dynamical behavior of a continuously-observed open quantum system [9, 12, 55].

A sophisticated theoretical basis for understanding such issues is presently maturing in the form of quantum trajectory theories [13 16], but significant technical challenges remain to be solved before definitive experiments can be performed in the lab. Our purpose in the present work is to report substantial progress towards surmounting such obstacles in the context of cavity QED, and hence towards achieving the essential experimental capabilities required to perform quantitative tests of measurement-based Stochastic Master Equations. We ultimately hope to be able to implement some recentlyproposed "applications" of the continuous observation of dissipative quantum dynamics, in fields such as quantum measurement [17.20], quantum chaos [18, 19], and quantum feedback control 21,20,23,24.

This article focuses on a detailed description of our recent experiments that record the complete time-evolution of interaction energy between one atom and a high-finesse optical cavity, during individual transit events of $\sim 250$ $\mu$ s duration. With characteristic atom-cavity interaction energies $E_{\text {int }} / \hbar \sim 10 \mathrm{MHz}$, we achieve measurement sensitivities $S_{g} \simeq 4.5 \mathrm{kHz} / \sqrt{\mathrm{Hz}}$ over a bandwidth that covers the dominant rates of variation in $E_{\text {int }}(1-$ $300 \mathrm{kHz}$ ). Unlike typical pump-probe measurements of scattering dynamics in real (e.g. diatomic) molecular systems [25,26], our experiments on the Jaynes-Cummings "molecule" yield a continuous time-domain record of the atom-cavity coupling during each individual "scattering" event (transit). The data clearly illustrate variations caused by atomic motion through the spatial structure of the cavity eigenmode and/or optical pumping among the atomic internal (Zeeman) states. In certain parameter regimes of the detuning and probe power, distinctive indications of the quantum-mechanical nature of the atom-cavity coupling can be seen in the photocurrent recorded from just a single atomic transit. For large $(\geq 50 \mathrm{MHz})$ atom-cavity detunings we obtain phasecontrast signals induced by individual atomic transits, corresponding to a regime of strong but dispersive coupling. Phase-quadrature measurements of atomic motion have been widely discussed in the quantum optics literature [27 29, 11, 24, 12 , but the present work provides the first experimental demonstration at the single-atom level. 
Because of the standing-wave spatial structure of the cavity eigenmode, and the corresponding rapid varation of the atom-cavity coupling strength over sub-wavelength distances, our data should in principle display a sensitivity of $1.5 \times 10^{-10} \mathrm{~m} / \sqrt{\mathrm{Hz}}$ to atomic displacements along the cavity axis. Unfortunately we cannot realize this figure as a precision for monitoring the atomic position, as we do not presently have any means of separating signal variations due to motion through the standing wave from "background" contributions due to transverse motion or optical pumping. In our concluding section, we shall briefly discuss our motivations for further work to disambiguate the nature of rapid variations in our data.

\section{BASIC THEORETICAL DESCRIPTION}

In simple terms, our experimental procedure is to drop a cloud of cold Cesium atoms from a magnetooptic trap (MOT) into a high-finesse optical cavity, while continuously monitoring the cavity's complex susceptibility with a weak probe laser 30,31. By limiting the number of atoms in the initial cloud, we can easily reach an operating regime in which atoms transit the cavity only one at a time. Using broadband heterodyne detection and a high-speed digitizer, we continuously record both the amplitude and phase of the transmitted probe beam during $\sim 50 \mathrm{~ms}$ time windows. Each window typically contains from zero to five atom transit signals.

The elementary theoretical description of such a measurement employs steady-state solutions of the nonselective Master Equation for a stationary two-level atom coupled to a single electromagnetic mode via the JaynesCummings interaction Hamiltonian. Atomic center-ofmass motion and optical pumping among Zeeman states can only be included in this treatment by allowing for a time-dependent atom-cavity coupling strength. Although this type of approach cannot make predictions about dynamical variations in the coupling strength, it does provides a quantitative basis for interpreting some time-independent features of our data. Our use of this "adiabatic" model may be justified to a certain extent by the separation of timescales that we achieve in optical cavity QED with laser-cooled atoms. In optimal cases, the atom-cavity coupling strength should vary by as little as a factor of $10^{-4}$ over the system damping time $\sim 30$ ns.

If we let $\rho$ denote the density operator for the joint state of the atom and cavity, the nonselective Master equation (in the electric dipole and rotating-wave approximations) reads:

$$
\begin{aligned}
\dot{\rho}= & \frac{-i}{\hbar}\left[\hat{H}_{0}, \rho\right]+\gamma_{\perp}\left(2 \hat{\sigma} \rho \hat{\sigma}^{\dagger}-\hat{\sigma}^{\dagger} \hat{\sigma} \rho-\rho \hat{\sigma}^{\dagger} \hat{\sigma}\right) \\
& +\left(\kappa_{a}+\kappa_{b}+\kappa_{c}\right)\left(2 \hat{a} \rho \hat{a}^{\dagger}-\hat{a}^{\dagger} \hat{a} \rho-\hat{a}^{\dagger} \hat{a} \rho\right), \\
\hat{H}_{0}= & \hbar \Delta \hat{a}^{\dagger} \hat{a}+\hbar \Theta \hat{\sigma}^{\dagger} \hat{\sigma}+\sqrt{2 \kappa_{a}} \mathcal{E}\left(\hat{a}+\hat{a}^{\dagger}\right)+H_{\mathrm{int}}, \\
H_{\mathrm{int}}= & \hbar g_{0} e^{-\left(y^{2}+z^{2}\right) / w^{2}} \cos \left(k_{L} x\right)\left[\hat{a} \hat{\sigma}^{\dagger}+\hat{a}^{\dagger} \hat{\sigma}\right] .
\end{aligned}
$$

Here $\gamma_{\perp}$ is the atomic dipole decay rate, $\kappa_{a}$ is the cavity field decay rate through the input mirror (through which the probe laser is injected), $\kappa_{b}$ is the cavity field decay rate through the output mirror, $\kappa_{c}$ is the cavity field decay rate due to intracavity scattering/absorption losses, $\Delta \equiv \nu_{a}-\nu_{p}$ is the atom-probe detuning, $\Theta \equiv \nu_{c}-\nu_{p}$ is the cavity-probe detuning, and the coupling strength $g_{0}$ is equal to half the maximum single-photon Rabi frequency. We treat the atomic center-of-mass coordinates $x, y, z$ as c-number parameters, with the $x$-axis conciding with the cavity axis and $z$ parallel to gravity. The Gaussian waist of our cavity mode is $w \simeq 45 \mu \mathrm{m}$. Note that we have written the Master Equation in a frame rotating at the drive frequency, so $\mathcal{E}$ is a constant term proportional to the complex amplitude of the driving field.

To find the steady-state density operator as a function of driving strength and various detunings, we simply set $\dot{\rho}_{s s}=0$ and solve for $\rho_{s s}$ using linear algebra. The expected amplitude and phase of the heterodyne photocurrent may then be computed as [32,14

$$
\left\langle i_{h e t}(t)\right\rangle=\eta f_{L}^{1 / 2} \sqrt{2 \kappa_{b}} \exp \left[i\left(\Omega_{L} t+\phi_{L}\right)\right] \operatorname{Tr}\left[\rho_{s s} \hat{a}\right],
$$

where $\eta$ represents the overall photodetection efficiency (including propagation losses between the cavity and photodetectors, heterodyne efficiency, and detector quantum efficiency), $f_{L}$ and $\phi_{L}$ represent the photon flux and phase of the (optical) local oscillator, and $\Omega_{L}$ is the frequency of the optical local oscillator relative to the rotating frame (cavity driving field). In the experiment, we mix $i_{\text {het }}(t)$ with an rf local oscillator at the frequency $\Omega_{L}$ (which ranges between 40 and $190 \mathrm{MHz}$ ) and separately record the in-phase and quadrature components of the slowly-varying envelope (with an analog bandwidth of $300 \mathrm{kHz}$ ).

Note that the presence of an intracavity atom can influence the heterodyne photocurrent only via the interaction Hamiltonian $H_{\text {int }} \propto \hbar g(\mathbf{r})$, where

$$
g(\mathbf{r}) \equiv g_{0} e^{-\left(y^{2}+z^{2}\right) / w^{2}} \cos \left(k_{L} x\right) .
$$

In a two-level approximation for the atomic internal dynamics, and for a classical treatment of the atomic centerof-mass degrees of freedom, all steady-state properties of the atom-cavity system are strictly determined by the value of $g(\mathbf{r})$ once the parameters $\left(\kappa_{a}, \kappa_{b}, \gamma_{\perp}, \Delta, \Theta, \mathcal{E}\right)$ have been specified. This includes the quantity $\left\langle H_{\text {int }}\right\rangle$, which represents one possible measure of the "interaction energy" between atom and cavity. Given that $g(\mathbf{r})$ appears to be a more fundamental measure of the interaction strength, however, we have adopted the convention $E_{\text {int }} \equiv \hbar g(\mathbf{r})$.

The atom-cavity interaction can be treated semiclassically using the optical bistability state equation (OBSE) [33. The OBSE is traditionally written in terms of the scaled field variables $x$ and $y$ (not to be confused with the atomic coordinates), with the correspondence

$$
x \equiv \frac{\langle\hat{a}\rangle}{\sqrt{m_{0}}}, \quad y \equiv \frac{\sqrt{2 \kappa_{a}} \mathcal{E}}{\left(\kappa_{a}+\kappa_{b}\right) \sqrt{m_{0}}},
$$


where the saturation photon number $m_{0}$ is given by $\frac{\gamma_{\perp}^{2}}{2 g^{2}}$. For a given driving strength $\mathcal{E}$, the expected intracavity field amplitude can be found by inverting the equation

$$
y=x\left(1+\frac{2 C}{1+\delta^{2}+x^{2}}+i \frac{\phi-2 C \delta}{1+\delta^{2}+x^{2}}\right),
$$

where we again work in a rotating frame at the drive frequency, $\delta \equiv\left(\omega_{a}-\omega_{p}\right) / \gamma_{\perp}$ represents the scaled atomprobe detuning, $\phi \equiv\left(\omega_{c}-\omega_{p}\right) /\left(\kappa_{a}+\kappa_{b}+\kappa_{c}\right)$ represents the scaled cavity-probe detuning, and the "cooperativity" $C$ is defined by

$$
C \equiv \frac{g^{2}}{2\left(\kappa_{a}+\kappa_{b}+\kappa_{c}\right) \gamma_{\perp}} .
$$

Note that the dependence of $g$ (and thereby $C$ and $m_{0}$ ) on the atomic position and internal state is implicit. A semiclassical prediction for the heterodyne photocurrent is obtained by substituting $x \sqrt{m_{0}}$ for $\operatorname{Tr}\left[\rho_{s s} \hat{a}\right]$ in equation (4).

\section{EXPERIMENTAL APPARATUS AND PROCEDURES}

Figure 1 provides a general overview of the apparatus, indicating the schematic arrangement of various components to be described below. The diode laser setup for forming the Cs MOT is not shown.

\section{A. High-finesse microcavity}

We use a Fabry-Perot high-finesse microcavity ("physics cavity") consisting of two spherical mirrors with $1 \mathrm{~m}$ radius of curvature [34]. The cavity was constructed with a mean length $l \simeq 107.5 \mu \mathrm{m}$, which we inferred from the cavity's measured free spectral range of $1.395 \times 10^{12} \mathrm{~Hz}$. The measured $l$ and specified radii of curvature geometrically determine the cavity's electromagnetic mode volume for $\mathrm{TEM}_{00}$ modes near $852 \mathrm{~nm}$ [35. Together with the dipole decay rate $\gamma_{\perp} / 2 \pi \simeq 2.6$ $\mathrm{MHz}$ for the Cs $6 \mathrm{P}_{3 / 2}$ level [36], this determines our optimal coupling constant $g_{0} / 2 \pi$ to be $\simeq 11 \mathrm{MHz}$ for $\sigma_{ \pm}$ transistions (specifically the $6 S_{1 / 2}\left(F=4, m_{F}= \pm 4\right) \rightarrow$ $\left.6 P_{3 / 2}\left(F=5, m_{F}= \pm 5\right)\right)$ and $\simeq 6 \mathrm{MHz}$ for $\pi$ transitions $\left(6 S_{1 / 2}\left(F=4, m_{F}=0\right) \rightarrow 6 P_{3 / 2}\left(F=5, m_{F}=0\right)\right)$ within the D2 Zeeman manifold [1, 37].

In order to allow cold atoms to fall into such a short cavity, we found it necessary to have the mirror manufacturer reduce the substrate diameters from the standard value of $7.75 \mathrm{~mm}$ down to $3 \mathrm{~mm}$ [34]. This reduced the "sagittal depth" of the curved mirror substrates and allowed us to maintain a gap of $\sim 100 \mu \mathrm{m}$ around the edge of the cavity. Machining of the mirror substrates was performed after they had been superpolished and coated, but this process did not seem to degrade the mirror reflectivities significantly. The nominal combined transmission and loss per mirror, before machining, was $1.5 \times 10^{-5}$. Direct measurements of the cavity finesse yield $\mathcal{F} \simeq 217,000$ at an optical wavelength of 852.36 $\mathrm{nm}$, consistent with a combined mirror transmission and loss $T^{*} \simeq 1.45 \times 10^{-5}$. This value of $T^{*}$ is inferred from the measured value of $l$ and the measured cavity HWHM $\kappa / 2 \pi \simeq 3.21 \mathrm{MHz}$.

The cavity used throughout the work described in this paper suffers from a rather pronounced birefringence, which for $\mathrm{TEM}_{00}$ modes near $852 \mathrm{~nm}$ induced a splitting of $8 \pm 2 \mathrm{MHz}$ between linearly-polarized eigenmodes. It is not entirely clear whether this birefrigence is a result of the substrate machining for diameter-reduction, a property of the coatings, or something associated with the mirror-mounting procedure used for this particular cavity. We note that recent efforts by other members of our group 31] have produced a cavity of approximately the same finesse with greatly-reduced birefringence (by a factor $\sim 25$ ), using mirrors from a different coating run and with great care taken to minimize cavity misalignments and stress on the mirror substrates.

Our two mirror substrates are mounted in vee-grooves atop independent aluminum blocks, with a piezoelectric actuator between the blocks for active servo-control of the mirror separation (cavity length). The cavity mount sits on a stack of alternating OFHC copper blocks and viton o-rings for passive vibration isolation, all within an ion-pumped vacuum chamber whose background pressure was typically $\sim 10^{-8}$ torr (inferred from the ion pump current). In order to bring the MOT as close as possible to the central axis of the physics cavity, we had to use a rather open (and non-magnetic) mount design, leading to some compromises in the way of mechanical stability. Sitting on the vibration-isolation stack and under vacuum, we found that the native noise spectrum of the cavity length extended out to about $4 \mathrm{kHz}$, with one prominent resonance at $50 \mathrm{~Hz}$ (which we attribute to a transmission resonance of the isolation stack). Due to a set of PZT-actuator resonances above $10 \mathrm{kHz}$, we have ultimately been limited to a unity-gain bandwidth $\simeq 1$ $\mathrm{kHz}$ for the cavity stabilization servo.

\section{B. Laser and cavity locking schemes}

Although the principle aim for this experiment is to stabilize the cavity length at some precise offset $\Delta / 2 \pi \sim$ $0-100 \mathrm{MHz}$ from the Cs D2 resonance at $852.359 \mathrm{~nm}$, the strong atom-cavity coupling places severe restrictions on the optical power that can be used for the purpose of generating an error signal. On resonance, the saturation intracavity photon number for our cavity is as small as $m_{0} \equiv \gamma_{\perp}^{2} / 2 g_{0}^{2} \simeq 0.1$, which sets a fiducial cavity throughput of $4 \pi \kappa_{b} m_{0} \sim 1 \mathrm{pW}$. With such low optical power it would be extremely difficult to obtain a high-quality error signal for locking the physics cavity. Other experiments 
performed in our group have circumvented this problem by using a chopped locking scheme, in which a strong "lock beam" alternates with a weak "probe beam" at $50 \%$ duty cycle and $\sim 1-4 \mathrm{kHz}$ frequency [38, 30, 31]. However, such a strategy inherently limits the servo unitygain bandwidths to $\sim 100 \mathrm{~Hz}$ at best, and would not be appropriate for future experiments with atoms trapped inside the cavity for long periods of time.

In this work we have developed an alternative locking scheme for the physics cavity, which employs an auxilliary diode laser at $836 \mathrm{~nm}$ to monitor the cavity length on a different longitudinal mode than that which couples strongly to intracavity Cs atoms. We use a commercial grating-stabilized diode produced by New Focus (Santa Clara, California). With a $16 \mathrm{~nm}$ detuning, we can send $\sim 40 \mathrm{nW}$ through the cavity and incur an AC Stark shift of only $\sim 60 \mathrm{kHz}$ for the atomic resonance at $\nu_{a}$. Using an EG\&G avalanche-photodiode/transimpedance amplifier module (model C30998) for AC detection of transmitted $836 \mathrm{~nm}$ light, we obtain an FM error signal (modulation frequency $3.8 \mathrm{MHz}$ ) with signal-to-noise ratio (SNR) $\sim 20$ at $30 \mathrm{kHz}$ bandwidth.

The $836 \mathrm{~nm}$ diode laser is stabilized to an auxilliary "transfer cavity," which consists of a pair of $25 \mathrm{~cm}$ radiusof-curvature mirrors at $\simeq 16 \mathrm{~cm}$ separation. One of the mirrors is mounted on a piezoelectric actuator to allow cancellation of DC drift and low-frequency noise. The transfer cavity has a linewidth $\simeq 100 \mathrm{kHz}$ at both $836 \mathrm{~nm}$ and $852 \mathrm{~nm}$, with an overall mode spacing $\sim 300 \mathrm{MHz}$. The transfer cavity is also used for pre-stabilization of the Ti:Sapphire laser, and some of the Ti:Sapphire light is used in a Cs modulation-transfer spectrometer [39] to provide an absolute reference for the transfer cavity length. From run to run we used one or two acoustooptic modulators to offset the Ti:Sapphire/transfer-cavity lock point by $+140,+87$, or $+43 \mathrm{MHz}$ relative to the Cs $6 \mathrm{~S}_{1 / 2}(F=4) \rightarrow 6 \mathrm{P}_{3 / 2}\left(F^{\prime}=5\right)$ transition.

By comparison of the Ti:Sapphire and diode laser error signals in their respective locks to the transfer cavity, we infer that the relative rms jitter between them is $\leq 10$ $\mathrm{kHz}$. For both laser locks we use the Pound-Drever-Hall technique [40] of detecting an FM signal in reflection from the tranfser cavity. The stability of the transfer cavity resonances with respect to atomic Cs lines in a vapor cell was such that we did not see any relative jitter beyond the measurement noise in our modulation-transfer spectrometer (SNR 50:1 in $30 \mathrm{kHz}$ bandwidth).

The Ti:Sapphire stabilization employs two feedback loops, one with $\sim 10 \mathrm{kHz}$ bandwidth to the tweeter inside the ring laser and another with $\sim 100 \mathrm{kHz}$ bandwidth to a VCO-driven, double-passed acoustooptic modulator $(\mathrm{AOM})$ just outside the laser cavity [41,42]. The mean frequency of the $\mathrm{AOM}$ is $76 \mathrm{MHz}$, and the error signal going to the VCO has a lower cutoff of $\sim 10 \mathrm{kHz}$ to prevent DC drifts. We note that use of the AOM is crucial for achieving high stability of the Ti:Sapphire frequency. The diode laser servo utilizes both feedback to the grating PZT and direct modulation of the injection current, achieving an overall unity-gain bandwidth $\sim 1 \mathrm{MHz}$.

Having locked both the diode laser and Ti:Sapphire to modes of the transfer cavity, which itself is locked to Cs, we use a travelling-wave electrooptic modulator to generate an rf sideband of the diode laser at $f_{0} \sim 200-$ $500 \mathrm{MHz}$. Either the upper or lower sideband is used to derive an FM error signal for locking the physics cavity by dithering $f_{0}$ at $3.8 \mathrm{MHz}$, thus allowing us to achieve arbitrary placement of the physics cavity mode near 852 $n m$ via the tunability of $f_{0}$.

\section{Evaluation of the physics-cavity servo}

Our basic requirement for the quality of the physicscavity servo was that relative jitter of the cavity resonance and the probe laser frequency should not contribute a significant amount of noise in the heterodyne photocurrent. Hence the relevant comparison to make is between the noise in both quadratures of a demodulated beatnote and the photocurrent fluctuations produced by the local oscillator alone. At $200 \mathrm{kHz}$ bandwidth and with a probe beam strength such that $m \equiv|\langle\hat{a}\rangle|^{2}=1.5$ in the empty cavity, the standard deviations of the phase and amplitude quadratures of the transmitted probe beam were measured to be 1.01 and 1.39 (respectively) relative to those of the local oscillator alone. Note that we have estimated the standard deviation of the quadratureamplitude signals produced by our optical local oscillator to be only a factor of 1.05 above the theoretical shot-noise limit (see below). We therefore believe that our overall excess noise factor $\beta \simeq 1.5$.

By taking some simultaneous recordings of the heterodyne photocurrent and the physics-cavity error signal, we were able to verify directly that the atomic transits do not affect the physics cavity servo.

\section{Cesium MOT}

To provide a source of cold Cs atoms, we used a standard magnetooptic trap loaded directly from a thermal beam [43]. Our choice of thermal-beam loading, as opposed to loading from a background vapor, was driven by an attempt to prevent accidental coating of the physics cavity mirrors with Cs. In more than two years of service, we did not detect any significant (> 5\%) change in the cavity finesse. Pre-cooling of the $\mathrm{Cs}$ beam was not necessary for this experiment, as we required only a very low rate of delivering single cold atoms into the cavity mode volume. Our MOT employs a six-beam configuration, and we orient the anti-Helmholtz coils for the trap so that their symmetry axis is parallel to that of the optical cavity. This leads to a MOT laser beam geometry with one beam axis running parallel to and just above the cavity, plus two beam axes in the plane of the mirror surfaces (Figure 2). 
The light for the MOT was provided by a pair of grating-stabilized diode lasers (SDL 5421-G2), one tuned to the $\mathrm{Cs} 6 \mathrm{~S}_{1 / 2}(F=4) \rightarrow 6 \mathrm{P}_{3 / 2}\left(F^{\prime}=5\right)$ cycling transition for trapping and the other to $6 \mathrm{~S}_{1 / 2}(F=$ $3) \rightarrow 6 \mathrm{P}_{3 / 2}\left(F^{\prime}=4\right)$ for repumping. Each trapping beam had $\sim 1 \mathrm{~cm}$ diameter and anywhere from $40 \mu \mathrm{W}$ to 4 $\mathrm{mW}$ of optical power, depending on how many atoms we were trying to send into the physics cavity. We typically used a Cs reservoir temperature of 60-80 C for the thermal beam, which effused through a $200 \mu$ pinhole and travelled an overall distance of $\sim 60 \mathrm{~cm}$ to the trapping region (with a cold mechanical collimator in the way to reduce loading of the ion pump). With an anti-Helmholtz field of around $25 \mathrm{G} / \mathrm{cm}$, we could load up to $\sim 2 \times 10^{5}$ atoms into a millimeter-sized cloud, whose mean temperature we estimate to be $\sim 100 \mu \mathrm{K}$ based on fluorescent imaging of free expansion. This temperature estimate is also supported by the spread in arrival times of individual atoms falling into the cavity.

When running the experiment we would load the MOT for about $0.5 \mathrm{~s}$, then drop it by quickly turning off the trapping beams with an AOM (using an rf switch with $\sim 45 \mathrm{~dB}$ attenuation). After the trapping beams were thus extinguished, we would ramp down the antiHelmholtz field according to an RC-filtered step with $\sim 4$ ms time constant. The repumping beam was left on all the time, so that falling atoms would be shelved in the $F=4$ ground hyperfine level before entering the cavity. No specific preparation was performed with respect to the atomic Zeeman states. Dropping $\sim 2 \times 10^{5}$ atoms we would generally see 30-50 atoms falling through the central part of the cavity mode volume, so for single-atom transit data we had to reduce the trapping beam power by a factor of 100 to reach 0-2 atoms per drop. The overall repitition rate for the trap-drop cycle was typically $0.6 \mathrm{~Hz}$.

\section{E. Probe generation and photodetection}

We used a balanced-heterodyne setup in order to achieve high-efficiency, zero-background photodetection of $\sim 1 \mathrm{pW}$ levels of $852 \mathrm{~nm}$ light transmitted through the physics cavity. The frequency difference between cavity probe light and the optical local oscillator for heterodyne detection was between 40-190 MHz, depending upon our choice for the atom-probe detuning. The probe light was generated from the Ti:Sapphire output by cascading a $+200 \mathrm{MHz}$ AOM and a tunable travelling-wave electrooptic modulator, which was driven between $-245 \mathrm{MHz}$ and $-440 \mathrm{MHz}$ to produce the desired atom-probe detuning. This indirect method was required to prevent contamination of the heterodyne photocurrent by electronic noise at the heterodyne frequency.

Light leaving the physics cavity first hit a colorseparation mirror which reflected $>99 \%$ of the $852 \mathrm{~nm}$ light but transmited $\approx 30 \%$ of the $836 \mathrm{~nm}$ light, allowing us to recover an error signal for locking the physics cavity (see above) without compromising the overall detection efficiency for the probe field. Residual $836 \mathrm{~nm}$ light going to the heterodyne setup amounted to only $\sim 30 \mathrm{nW}$ and had negligible effect on the photocurrent of interest.

The local oscillator (LO) for the optical heterodyne was spatially cleaned by a $\mathcal{F} \sim 1000$ Fabry-Perot cavity (linewidth $\sim 1 \mathrm{MHz}$ ), which also served to strip off spectral noise at $76 \mathrm{MHz}$ associated with the AOM servo for stabilization of the Ti:Sapphire frequency. The cleaning cavity was locked using the Pound-Drever-Hall method [40] with FM sidebands at $24 \mathrm{MHz}$, which likewise had to be kept weak in order not to saturate the $\mathrm{AC}$ gain of the heterodyne photodetectors. We used a total of $\sim 2$ $\mathrm{mW}$ in the $\mathrm{LO}$, which generated a shot-noise level $\sim 5$ $\mathrm{dB}$ above the electronic noise of the photodetectors in the frequency range of interest.

The difference photocurrent from the balanced heterodyne detectors was amplified up to $-50 \mathrm{dBm}$ or higher, then divided by a $0^{\circ} \mathrm{rf}$ splitter. An independent signal generator was used to produce an rf local oscillator at the heterodyne frequency, and it was halved using a $90^{\circ}$ rf splitter. The two identical copies of the photocurrent were mixed with the in-phase and quadrature copies of the rf LO to produce an orthogonal pair of quadrature amplitude (QA) signals at baseband. The QA signals were further amplified, and passed through $300 \mathrm{kHz}$ ana$\log$ filters with a roll-off of $12 \mathrm{~dB} /$ octave. We used a 12-bit ADC to sample both QA's simultaneously at a rate of $10 \mathrm{MHz}$ per channel, which is sufficiently high to avoid signal aliasing completely. Following each drop of the trap, we continuously recorded both QA's for a data acquisition window of $50 \mathrm{~ms}$ and streamed the data to a hard drive for offline processing following the experimental run.

Ideally, we would like the data acquisition procedure just described to yield directly the amplitude and phase quadrature amplitudes of light transmitted through the cavity. If we write the transmitted optical field as $\mathcal{A}(t) e^{-i \omega_{p} t}$, where $\mathcal{A}(t)$ is a slowly-varying complex amplitude, the amplitude $x_{a}$ and phase $x_{p}$ quadratureamplitudes are defined by $\mathcal{A} \equiv x_{a}+i x_{p}$. With respect to the quantum-mechanical theory of the Master Equation (11), $\mathcal{A} \propto\langle\hat{a}\rangle$. Note that we define $\mathcal{A}$ to have zero phase when the cavity is empty, so that $x_{p}$ should have zero mean when there are no intracavity atoms.

Given the way that we generate the probe beam, however, we have no way of generating a phase-locked rf local oscillator to recover $x_{a}$ and $x_{p}$ directly. The phase of the heterodyne photocurrent differs from the phase that the light has just after it leaves the cavity because of fluctuations in the relative optical path length travelled by the signal beam and optical local oscilator in reaching the photodetectors. So the two signals produced by mixing the photocurrent with the shifted and unshifted copies of our rf local oscillator correspond to an orthognal, but rotated pair of quadrature amplitudes $x_{1}, x_{2}$ : 


$$
\left(\begin{array}{l}
x_{1} \\
x_{2}
\end{array}\right)=\left(\begin{array}{cc}
\cos \phi & -\sin \phi \\
\sin \phi & \cos \phi
\end{array}\right)\left(\begin{array}{l}
x_{a} \\
x_{p}
\end{array}\right) .
$$

Luckily, the characteristic timescales for fluctuations in the phase $\phi$ are quite long $(\sim 10-100 \mathrm{~ms}$, corresponding to acoustic disturbances) compared to the $250 \mu$ s duration of an individual atom-transit signal. In processing the recorded data to produce the plots discussed below, we have therefore used an "adaptive" definition of the amplitude and phase quadrature amplitudes. Within a window of $2 \mathrm{~ms}$ preceding the signal of interest, we estimate the instantaneous value of $\phi$ by determining the rotation of $x_{1}, x_{2}$ that produces one quadrature $\tilde{x}_{p}$ with zero mean and one quadrature $\tilde{x}_{a}$ with positive mean. Then $\tilde{x}_{p}$ is operationally defined to be the phase quadrature photocurrent, and $\tilde{x}_{a}$ is the amplitude quadrature photocurrent.

Figure (3) shows an example of a $15 \mathrm{~ms}$ segment of our quadrature amplitude data. Note that some excess lowfrequency noise can still be seen in the phase quadrature, which constrains the lower end of our measurement bandwidth to $\sim 1 \mathrm{kHz}$. Six prominent atom-transit signals, characterized by a sharp drop in the amplitude quadrature and a simultaneous increase in the phase quadrature, can be seen between $t=0.009 \mathrm{sec}$ and $t=0.013 \mathrm{sec}$. In our subsequent discussions of the data, we shall focus on individual signal "events" of this type.

\section{DETERMINATION OF INTRACAVITY PHOTON NUMBER}

In order to make quantitative comparisons between data and theory, we need to calibrate the strength of the driving field used in each experimental run. As we will ultimately choose to ignore DC optical phase offsets, the relevant quantity for us will be the number of photons $m$ that builds up when the cavity is empty. This corresponds to $2 \kappa_{a}|\mathcal{E}|^{2} /\left(\kappa_{a}+\kappa_{b}+\kappa_{c}\right)^{2}$ in the Master Equation case and $|y|^{2} / m_{0}$ for the OBSE.

Our strategy for determining the intracavity photon number during experimental runs has been to work backwards from the heterodyne photocurrent observed with no atoms in the cavity, using the expression 32

$$
\left(\frac{S^{2}}{N}\right)_{h e t}=4 T \eta \kappa_{b} m
$$

where $S$ is the mean value of the demodulated amplitude quadrature signal (i.e. the heterodyne photocurrent is of the form $S \cos (\omega t+\phi)), N$ is the mean-squared power of fluctuations in the same signal due to (optical) shot noise, $T$ is the measurement interval, and $m \equiv|\langle\hat{a}\rangle|^{2}$. Note that the above expression is valid for a coherent signal beam, which we assume to be the state of the light transmitted through the cavity when no atoms are present. For an accurate calibration, we thus need to know the output mirror transmission $\kappa_{b}$, the total cavity loss (which can be determined from measurements of the cavity finesse), and the overall photodetection efficiency $\eta$. In determining $m$ from the data, we typically chose $T$ on the order of $1 \mathrm{~ms}$. The following subsections provide further detail for each critical aspect of the calibration.

\section{A. Evaluation of local oscillator noise}

Ideally, the quantity $N$ appearing in equation (10) should coincide with the photocurrent noise power $n$ observed when the signal beam is blocked. This would allow us to determine the intracavity photon number without having to calibrate the exact gains of the photocurrent amplifiers, etc. But the procedure is invalid if the optical local oscillator carries excessive technical noise, so we have empirically checked the scaling of our nominal $N$ with the DC optical power of the LO. A fit of the data to the functional form $n=a P+b P^{2}$ yields $b / a \simeq 0.11$ $\mathrm{mW}^{-1}$. Given our typical operating LO power of $1 \mathrm{~mW}$ per detector, and considering the relative magnitudes of other uncertainties, we approximate $N \simeq n$. Note that the LO does pass through an intensity stabilizer with $\sim 400 \mathrm{~Hz}$ bandwidth just before entering the cleaning cavity.

\section{B. Measurement of cavity decay rates}

We directly determined the total cavity field decay rate $\kappa_{a}+\kappa_{b}+\kappa_{c}$ by making a calibrated measurement of the HWHM of a cavity $\mathrm{TEM}_{00}$ resonance. In order to minimize systematic errors, we did this by using the heterodyne detectors to monitor the transmitted optical power of a fixed-frequency Ti:Sapph probe beam while scanning the cavity length. The cavity length was always under servocontrol during the measurement, as we generated the scan by stepping the frequency of the rf going to the travelling wave modulator for the locking diode laser. The total cavity field decay rate is then given by the resonance HWHM measured in terms of the modulator rf scan, times a correction factor of the ratio of the diode laser and Ti:Sapph wavelengths. We find $\kappa_{a}+\kappa_{b}+\kappa_{c} \simeq 3.2 \mathrm{MHz}$.

As the mirrors used to construct the cavity should be identical, we assign half the total losses to each mirror. Unfortunately, we did not manage to characterize the ratio of intracavity losses $\kappa_{c}$ to transmission losses $\kappa_{a}+\kappa_{b}$ before we accidentaly damaged the cavity. Given that the mirror coatings we have previously received from the same manufacturer have displayed very low scattering/absorption loss $\left(\sim 1.1 \times 10^{-6}\right)$ in the wavelength range of interest 45, we have assumed $\kappa_{b}=1.6 \mathrm{MHz}$. 


\section{Measurement of $\eta$}

Three principal factors determine the value of $\eta \equiv \mathcal{V} \varepsilon \tau$ : the spatial overlap $\sqrt{\mathcal{V}}$ between the signal beam and the optical local oscillator, the photodetector quantum efficiency $\varepsilon$, and the (power) efficiency $\tau$ with which we transfer light from the cavity ouput to the photodetectors. Using an optical powermeter we measured $\tau=0.9$, as well as $\varepsilon \simeq(0.68,0.71)$ for our two photodetectors. We measured $\mathcal{V} \simeq 0.65$ by producing a DC fringe between the signal beam and the optical local oscillator, with both beams adjusted to a power level of $1 \mu \mathrm{W}$. The power adjustments were made upstream of the physics cavity and LO cleaning cavity, ensuring that no optical misalignments were introduced in the process. We obtained an independent measurement of $\mathcal{V} \varepsilon \simeq 0.4 \pm 0.05$ from the heterodyne signal-to-noise ratio obtained with a measured signal power of $10 \mathrm{nW}$. Note that the reasonable agreement between this latter value and the directlymeasured $\mathcal{V} \varepsilon$ provides further indication that our optical local oscillator bears minimal excess noise, and that our assumptions $\kappa_{b}=\kappa_{a}, \kappa_{c} \sim 0$ are valid.

Although it could have been avoided, we did suffer one additional loss of detection efficiency due to gain and phase imbalance in the subtraction of heterodyne photocurrents. Several weeks after we took the data sets shown below, we realized that one photodetector output had an rf signal $4 \mathrm{~dB}$ higher than the other (this figure includes both the deviation of our heterodyne beamsplitter from being 50/50, the difference in photodiode quantum efficiencies, and the difference in transimpedance gains), and a phase offset of 1 radian. If we write the two photocurrents (for a coherent signal beam) as $i_{1}=\frac{1}{\sqrt{2}} e^{-i \omega t}+\xi_{1}$ and $i_{2}=\frac{-g}{\sqrt{2}} e^{-i \omega t+i \phi}+g \xi_{2}$ (where $\xi_{1}$ and $\xi_{2}$ are uncorrelated gaussian white noises with zero mean and unit variance), the difference photocurrent is

$$
i_{-}=\frac{1+g e^{i \phi}}{\sqrt{2}} e^{-i \omega t}+\sqrt{1+g^{2}} \xi,
$$

where $\xi$ is again a gaussian white noise process with zero mean and unit variance. The complex imbalance $g e^{i \phi}$ should thus reduce the effective photodetection efficiency (for the purpose of evaluating the heterodyne signal-tonoise ratio (100) by

$$
\eta \rightarrow \frac{1}{2} \eta \frac{\left|1+g e^{i \phi}\right|^{2}}{1+g^{2}} .
$$

Using the measured $g \simeq 0.63$ and $\phi \simeq 0.85 \mathrm{rad}, \eta \rightarrow 0.8 \eta$, and we quote an overall value of $\eta \simeq 0.32$.

\section{NUMERICAL SIMULATIONS}

In order to facilitate the interpretation of our data, we generated some rudimentary Monte Carlo simulations of the heterodyne signals that we should see as atoms fall through the cavity 1 Our code simulates threedimensional, classical center-of-mass motion of individual atoms under the influence of fluctuating forces due to their strong interactions with the cavity field. Gravity is also included in the kinematics, but plays only a minor role over the $\sim 1 \mathrm{~ms}$ duration of the simulations.

\section{A. Overall scheme for the simulations}

The basic scheme of the simulations is to precompute the values of the mean cavity-induced force, the cavityfield-induced diffusion coefficient, and the steady-state (complex) amplitude for the intracavity field as a function of atomic position. Hence, every other degree of freedom in the simulation is adiabatically eliminated and slaved to the atomic motion, which is assumed to be the slowest and the "stiffest" process in the dynamics. The mean force and intracavity field are derived from steadystate solutions of the Master Equation (11), and the cavity diffusion is computed using the Quantum Regression Theorem [46]. In each timestep, the code first performs an interpolation on the precomputed tables of values to determine the appropriate change in atomic momentum (which includes a stochastic increment consistent with the local value of the diffusion constant), records an appropriate value for the cavity output field, and then updates the atomic position and momentum. The simplest possible integration scheme is used (explicit Ito-Euler), yielding order 0.5 convergence in the timestep. We chose a timestep of $7.5 \mathrm{~ns}$ in order to keep the run-times for the simulations reasonable under Matlab on our Pentium II workstations, and this should have been sufficiently small to keep the integration error below other sources of inaccuracy.

The principle shortcoming of this scheme is that it assumes the atomic velocity will remain small enough that variations in the coupling strength $g$ will be negligible over timescales on the order of $\kappa^{-1}$ and $\gamma^{-1}$. If this condition is violated, then the steady-state values of the mean cavity force and cavity output are no longer appropriate. Another major approximation was made in treating only a two-level atom - optical pumping and/or the associated opto-mechanical effects (e.g., spontaneous forces) could certainly play an important role in determining the shapes of our observed transit signals. A third, less severe approximation is that we have treated the stochastic process associated with recoil from spontaneous-emissions as being statistically independent from the one associated with dipole-force fluctuations. The possibility that interesting effects could arise due to quantization of the atomic motion is of course ignored as well.

\footnotetext{
${ }^{1}$ The computational work described in this section was conducted by H.M..
} 
Given the relative simplicity of our simulation scheme, it would be inappropriate to draw any strong conclusions about our experimental data soley on the basis of apparent similarities with the numerical results. However, the simulations do provide an appealing model for the atomic center-of-mass dynamics and associated heterodyne signals, with predictions that seem to be fully consistent with what we see in the actual experiment. This model suggests some interesting interpretations for qualitative features of the data. For example, the marked asymmetry (in time) of most of our experimental transit signals may be associated with an initial phase of atomic "channeling" in the dipole potential provided by the intracavity optical field, followed by a sudden escape from local confinement due to cavity-field-induced momentum diffusion. Such a dynamical process, if it could be confirmed in some way, would bear a strong resemblence to the Levy Walk behavior predicted in [47,48] and observed in 49. Our simulations also suggest that isolated dips and steps in the observed transit signals may be associated with sudden changes in atomic localization relative to the cavity standing wave.

Before presenting some results of the atomic-motion simulations, let us first describe the numerical method used to compute the cavity diffusion coefficients.

\section{B. Computation of diffusion coefficients}

Following Doherty et al [50], we computed the diffusion coefficient associated with dipole-force fluctuations according to

$$
D=\lim _{t \rightarrow \infty} \operatorname{Re} \int_{0}^{\infty}\langle\mathbf{f}(t), \mathbf{f}(t-\tau)\rangle d \tau,
$$

where $\mathbf{f}$ is the force operator

$$
\mathbf{f}=-i \hbar \nabla g(\mathbf{r})\left(\hat{a}^{\dagger} \hat{\sigma}_{-}-\hat{a} \hat{\sigma}_{+}\right)
$$

and we define

$$
g(\mathbf{r}) \equiv g_{0} \cos \left(k_{L} x\right) \exp \left[-\left(y^{2}+z^{2}\right) / w^{2}\right]
$$

Note that the vector nature of the force operator comes only from the gradient $\nabla g(\mathbf{r})$. Unlike the computation of mean forces and the expected intracavity field amplitude, the evaluation of (13) requires an actual time-integration of the cavity-QED Master Equation (11). Note that

$$
\langle\mathbf{f}(t), \mathbf{f}(t-\tau)\rangle \equiv \operatorname{Tr}\left[\mathbf{f} e^{\mathcal{L} \tau} \mathbf{f} \rho_{s s}\right]-\langle\mathbf{f}\rangle^{2},
$$

where $\mathcal{L}$ is the Liouville superoperator defined by

$$
\frac{d}{d t} \rho=\mathcal{L} \rho
$$

with reference to the cavity-QED Master Equation (11). Knowing the steady-state atom-cavity density matrix $\rho_{s s}$, one can evaluate the correlation function in (16) by integrating the Master Equation for a time $\tau$ with $\mathbf{f} \rho_{s s}$ as an initial condition, multiplying the result by $\rho_{s s}$, and finally taking the trace.

The numerical integration was performed by a $\mathrm{C}++/$ MPI code on an SGI/Cray Origin-2000 cluster. We used a truncated basis of 25 Fock states for the cavity mode, using an explicit Euler integration with a 1 ps timestep and $5 \mu$ s total integration time.

Figure 1 shows the results of such a calculation for the diffusion coefficient as a function of atomic position along the standing wave, for an atom located on the cavity axis. Recall that the cavity mode function varies as $\cos \left(k_{L} x\right)$ along the cavity axis, and as a gaussian in the transverse dimensions. Starting from the top, the four curves represent $\Delta=0,10,30$, and $50 \mathrm{MHz}$, all with a probe strength such that an average of 2 photons would build up in the cavity if it were empty and with $\Theta=0$. Figure 5 shows similar results, all for $\Delta=50 \mathrm{MHz}$, but with an average intracavity photon number (for the empty cavity) of $m=8$ for the top curve, 4 for the middle, and 2 for the lowest. As the variation of $g$ along the standing wave is much more rapid than in the transverse (Gaussian) directions, we approximated $\nabla g(\mathbf{r}) \simeq d g / d x$ in expression (13). The computation of $\mathbf{f}$ for the mean force field was of course three-dimensional.

Note that expression (13) gives the diffusion coefficient associated with dipole force fluctuations only - it takes no account of recoils from spontaneous emission. We therefore incorporate a second diffusion process in the simulations, whose coefficient is computed from the expectation value of the atomic excitation at each point in space:

$$
D_{r e c}=\frac{\left(\hbar k_{L}\right)^{2}}{25} \Gamma\left\langle\hat{\sigma}_{+} \hat{\sigma}_{-}\right\rangle .
$$

Here $\Gamma$ is the atomic spontaneous emission rate (Einstein A coefficient), and the number $1 / 25$ comes from averaging over the angular distribution pattern for dipole radiation [50].

\section{Discussion of results}

Figure 6 shows an example of the atomic position along the standing-wave $x$ and the atomic velocity along the standing-wave $v_{x}$ from one typical simulation. Also shown are the mean value of the intracavity phase quadrature amplitude $\left\langle q_{p}\right\rangle \equiv i\left\langle\hat{a}-\hat{a}^{\dagger}\right\rangle / 2$, and a simulated heterodyne signal with Gaussian noise added at the level appropriate to our experimental parameters (see equation (10)). Note that the quantity $\left(\left\langle q_{p}\right\rangle\right.$ plus noise) is proportional to the photocurrent $\tilde{x}_{p}$ that would be recorded in our experiment. In the bottom row of the figure, $\left\langle q_{p}\right\rangle$ and $\left\langle q_{p}\right\rangle$ plus shot-noise are shown at an ana$\log$ bandwidth $300 \mathrm{kHz}$ in order to illustrate the effects of finite detection bandwidth on the qualitative features of the data [50]. The results clearly suggests that the gross features of the simulated signals are most strongly 
affected by atomic motion along the standing wave, in that the overall gaussian profile associated with motion in the $z$-direction becomes distorted by the "envelope" of the oscillatory variations due to motion along $x$ (recall $\left.g(\mathbf{r})=\cos \left(k_{L} x\right) \exp \left[-\left(y^{2}+z^{2}\right) / w^{2}\right]\right)$.

Looking at the results for $x(t)$ and $v_{x}(t)$, we see that the atom in this particular simulation was mechanically confined within one well of the cavity standing wave until $t \sim 810 \mu \mathrm{s}$. That is, $x$ did not vary by more than $\lambda / 4$ and $v_{x}$ displays the oscillations that one would expect to see for an atom trapped within a potential well. At time $t \sim 810 \mu \mathrm{s}$, however, we see that momentum diffusion finally pushes $v_{x}$ above some threshold value such that the atom is able to escape from local confinement and "fly above" the periodic dipole potential associated with the cavity standing wave. Generally speaking, one expects this type of escape to occur as the atom passes through the cavity axis in the $-z$-direction and the standing-wave potential wells begin to decrease in depth. The expectation value $\left\langle q_{p}\right\rangle$ clearly reflects the qualitative features of the atomic trajectory $x(t)$, although we also see that the details are lost in the measured signal due to heterodyne shot-noise. What survives in the simulated heterodyne signal is an overall asymmetry in the envelope of the transit-signal, with a sudden "step" downwards at $t \sim 800 \mu \mathrm{s}$ (as suggested by the dotted vertical line).

Figure 7 shows four simulated signals with $\Delta=10$ $\mathrm{MHz}$ and an average intracavity photon number of $m=$ 2 , and two simulated signals with $\Delta=50 \mathrm{MHz}$ and $m=4(\Theta=0$ in all cases $)$. In each subplot, the upper trace shows the intracavity amplitude quadrature $\left\langle q_{a}\right\rangle \equiv\left\langle\hat{a}+\hat{a}^{\dagger}\right\rangle / 2$ versus time, and the lower trace (with zero mean value) shows the intracavity phase quadrature $\left\langle q_{a}\right\rangle$ versus time. Again, Gaussian noise has been added to the simulated signals in order to reproduce the overall signal-to-noise ratio predicted by equation (10). In all simulations, the atom was started at a position 7 gaussian waists above the cavity with a vertical velocity of $-47 \mathrm{~cm} / \mathrm{s}$. The initial transverse position, transverse velocity, position along the cavity standing-wave axis, and velocity along the standing-wave were randomly assigned within parameter ranges that were narrow enough to ensure that most simulated atom-drops produced a sizeable signal.

The simulated signals in Figure 7 may be compared directly to the real data shown in Figure 8. Like the simulation shown in Figure 6, the signals in Figure 7 display a generic asymmetry and often contain an isolated dip or step. The features of this type that are marked by an arrow near the horizontal axis are again associated with sudden changes in the atomic localization relative to the cavity standing wave (as determined by examining $x(t)$ from the simulations).

Although we have the benefit of knowing both the "actual" trajectory of an atom and the corresponding heterodyne signal in our simulations, a priori knowledge of the atomic trajectory is of course unavailable in our experiment. Hence, the results of our numerical simulations can only provide general guidelines for how we might try to infer something about individual atomic trajectories from our heterodyne transit signals. First of all, it appears that signficant asymmetries in the observed signals could provide evidence that we do in fact observe mechanical aspects of the atom-cavity coupling within individual atomic transits. Relative to the approximations involved in our simulations however, one should bear in mind that such asymmetries could also arise from optical pumping processes that may occur during at atomic transit through the cavity. Second, the simulations suggest that the transit signal envelopes are most strongly influenced by atomic motion along the cavity standing wave, as opposed to details of the motion along $y$ or $z$. Third, isolated dips or steps in the signal could be indicative of sudden changes in the atomic localization with respect to the standing wave. Again however, we should be careful to note that such features might also be caused by intracavity optical pumping.

\section{DATA}

Moving on to the experimental data, let us first discuss some transit signals displayed in the same fashion as the simulations of Figure 6 . The quality of this data illustrates our experimental ability to perform continuous, nearly quantum-noise limited measurement of the atom-cavity interaction energy during individual scattering events. In the second subsection we shall display transit data on the complex plane, and present a quantitative comparison to theoretical predictions of the atom-cavity system's complex optical susceptibility. We shall see that in optimal cases, sufficient information may be obtained from an individual atom-transit signal to distinguish unambiguously between quantum and semi-classical models of cavity QED.

Note that all of the data shown here were taken with cavity-probe detuning $\Theta \equiv \nu_{c}-\nu_{p}=0$, while the atomprobe detuning $\Delta \equiv \nu_{a}-\nu_{p}$ and probe power $m$ are varied.

\section{A. High bandwidth single-atom transits}

The basic unit of our experimental data is a continuous stream of quadrature-amplitude values versus time, recorded for $\sim 50 \mathrm{~ms}$ windows following each dropping of the MOT. One $\sim 15 \mathrm{~ms}$ segment of such data is shown in Figure 3. This particular data segment was taken with detunings $(\Delta=10, \Theta=0) \mathrm{MHz}$ and with a probe power such that an average of 1.5 photons would build up in the cavity if it were empty $(m=1.5)$.

Figure 8 shows six of the largest single-atom transit signals from our entire data set, which covers detunings $\Delta$ from -10 to $+100 \mathrm{MHz}$ and probe powers $m$ from 1.5 to 11 photons. The particular values of $\Delta$ and $m$ for each 
signal in Figure 8 are displayed by the y-axis. The data are shown at our full analog bandwidth of $300 \mathrm{kHz}$ and sampling rate of $10 \mathrm{MHz}$ (12 bit resolution). Note that we have displaced the amplitude quadrature signals $\tilde{x}_{a}$ by +400 in order to prevent them from overlapping with the phase quadrature signals $\tilde{x}_{p}$.

We have found that transit signals of maximal contrast tend not to have very much internal structure, although there is a fairly prominant dip at the point indicated by an arrow in subplot "dat4," a set of three dips indicated by the arrow in subplot "dat6," and oscillatory structure in the signal of subplot "dat5." The signal of subplot "dat4" is really not so different in its overall structure than the simulations shown in Figure 6 and in the "traj10" subplot of Figure 7. One can see that the shapes of the overall signal envelopes do vary substantially and are generally asymmetric in time. The distribution of the atom-transit signal between amplitude and phase quadratures clearly depends upon the probe detuning, as will be discussed in greater detail below. For the data event recorded with $\Delta=0$, one sees that there is only a reduction in the power of the transmitted probe without any shift in its phase. At all other detunings, the atom-induced reduction of amplitude quadrature is partly offset by an increase in the phase quadrature, indicating a significant phase shift of the transmitted probe beam. In subplot "dat6" taken with $\Delta=50 \mathrm{MHz}$, the signal is seen to primarly reside in the phase quadrature.

For signals such as those shown in Figure 8, we may estimate a full-signal to rms-noise ratio of approximately 2.5 for the phase quadrature and 4 for the amplitude quadrature. Combining the two signals, we have an overall ratio of 4.5. The signal bandwidth is $300 \mathrm{kHz}$, implying a relative sensitivity of $0.22 / \sqrt{3 \times 10^{5}} \simeq 4 \times 10^{-4}$ $\mathrm{Hz}^{-1 / 2}$. Assuming that the largest signals correspond to atoms reaching the maximal coupling strength of $g_{0}=11$ $\mathrm{MHz}$, this sets our broadband sensitivity $S_{g}$ to timevarations in $g \equiv E_{\text {int }} / \hbar$ to be $S_{g} \simeq 4.5 \mathrm{kHz} / \sqrt{\mathrm{Hz}}$. Given our overall photodetection efficiency $\eta \simeq 0.32$ and our independent assesment of the excess noise factor $\beta \simeq 1.5$ (corresponding to technical noise on the optical local oscillator power and on the physics cavity lock), we find that this value lies only a factor of $\beta / \sqrt{\eta} \simeq 2.7$ above the fundamental quantum noise floor. We maintain this high sensitivity over 2.5 decades of signal frequency, from our full photodetection bandwidth of $300 \mathrm{kHz}$ down to $1 \mathrm{kHz}$ (at which point we are limited by residual technical noise in our measurements of the phase quadrature).

Although a complete theoretical description of our experiment at the level of a Stochastic Master Equation [14 could in principle be formulated to include three-dimensional quantized atomic center-of-mass motion, cavity birefringence, and the full manifold of internal atomic Zeeman states, the resulting model would almost certainly be too complex to be useful for quantitative interpretations of our existing data. Nevertheless, we wish here to stress that only a theory that ex- plicitly treats the quantum conditioning [13 15] of the atom-cavity evolution on the stochastic component of the heterodyne photocurrent could provide a full account of the statistics and autocorrelation properties of our atomtransit data. In this crucial sense, we would claim that our recent work may be distinguished from all other efforts in contemporary experimental physics. Of course, much remains to be done before we can offer definitive proof, so our continuing efforts will largely be directed towards the unambiguous demonstration of quantum conditional dynamics in cavity QED.

\section{B. Transit phasors}

In addition to displaying our atom-transit signals in the format of photocurrent versus time, we can also construct parametric plots of $\tilde{x}_{a}$ versus $\tilde{x}_{p}$ to examine the correlation induced between these two quantities by the atom-cavity interaction. This is equivalent to compiling a histogram of the complex amplitude of the optical field transmitted through the cavity during an atomic transit, and hence to a continuous monitoring of the atom-cavity system's complex optical susceptibility. The underlying probability distributions for such histograms are determined by the (evolving) Husimi Q-function for the intracavity field [32, 14].

Many of our measurements have been conducted in a regime of strong cavity-driving fields $\left(m>m_{0} g_{0}^{2} / \Delta^{2}\right)$, with the consequence that significant effects of saturation and optical nonlinearity can be seen in our experimental data. As has been extensively discussed in the cavity-QED literature, the nonlinear optical response of the atom-cavity system represents an important experimental signature that may be used to distinguish between quantum and semiclassical theoretical models for the atom-cavity interaction. Prior to this work, four groups [51 53:31 have reported nonlinear measurements in cavity QED. In each of these previous experiments, the information gained per atomic transit about the details of the system's nonlinear response was far less than in the data we shall present here. Hence, we wish again to stress that the techniques developed in our most recent experimental work have opened new possibilities for exploring quantum dynamics at the level of single quantum realizations (trajectories), as opposed to the level of ensemble averages.

In Figures 9 and 10, we display single-transit data on the complex plane. The experimental data are displayed as gray dots, with each dot representing the values of $\tilde{x}_{a}$ and $\tilde{x}_{p}$ at some particular time during an atomic transit. In order to produce a set of dots from a transit signal such as those shown in Figure 8, the quadrature amplitude signals were first passed through an anti-aliasing filter and then resampled every $10 \mu$ s to produce a discrete set of points $\left\{\tilde{x}_{a}^{i}, \tilde{x}_{p}^{i}\right\}$. For every $i$, a gray dot is placed at radius $\sqrt{\left(\tilde{x}_{a}^{i}\right)^{2}+\left(\tilde{x}_{p}^{i}\right)^{2}}$ and polar angle $\tan ^{-1}\left(\tilde{x}_{p}^{i} / \tilde{x}_{a}^{i}\right)$. 
These transit phasors represent a simple way of looking at the correlation between the amplitude and phase of light transmitted through the cavity, with time removed from the picture. We believe that this type of plot is the best format for comparisons to theory, because atomic internal (optical pumping among Zeeman states) and external (motion through the cavity eigenmode) dynamics should be factored out. In the simplest approximation, these two types of processes merely induce fluctuations in the atom-cavity coupling $g$, which should only move the locus of our gray dots in and out along a curve parametrized by $g$. The overall shape of this curve should be dictated by the interaction Hamiltonian for the atom and cavity mode, and is therefore quite easy to compute.

In Figure 9, we show a set of transit phasors taken at various probe detunings. The data overlay theoretical curves predicted by the quantum and semiclassical theories. The quantum-mechanical predictions are computed by finding the steady-state solution of the Master Equation (11) for values of $g$ in the range $\left[0, g_{0}\right]$ and appropriate values of $\Delta$ and $m$. The solid curves represent an interpolation through the discrete set of computed values for $\left\langle q_{a}\right\rangle$ and $\left\langle q_{p}\right\rangle$ as a function of $g$. The semiclassical prediction is computed in the analogous manner, using the Optical Bistability State Equation (7) rather than the quantum Master Equation. Note that both curves must agree for $g=0$, and this common point of origin is marked in each subplot by a triangle. The $g=g_{0}$ endpoint of the quantum curve is marked by a circle, and the $g=g_{0}$ endpoint of the semiclassical curve is marked by an $\times$. In the subplots of Figure 9, the grey dots represent an overlay of data from two individual atom-transit signals. The two particular signals displayed in each subplot were selected on the basis of having maximal "contrast" among all the signals from a given data set, under the assumption that the atoms causing maximal signals should have come the closest to actually achieving $g=g_{0}$ on their way through the cavity. Quantum theory is seen to predict the observations quite well for all the detunings shown in Figure 9.

The quantum and semiclassical predictions shown in Figure 9 do not differ significantly except for the case of $\Delta=0$. Generally speaking, the two theories are known to agree in their predictions for weak-field response of the atom-cavity system but to differ in their predictions regarding saturation of the optical response. We here present experimental evidence from single atom transits for the quantum character of saturation in the atomcavity system's response to near-resonant driving fields.

In Figure 10 we show a set of transit phasors for fixed detuning $\Delta=10$ but variable probe strength $m=2.8$, $4.4,7$, and 11. A clear discrepancy between the quantum and semiclassical theory may be seen in this sequence of plots, with the data (gray dots) showing significant preference for the quantum-mechanical predictions. Each subplot in Figure 10 represents an overlay of data from three individual atom-transit signals, in order to fill in the overall "shape" of the experimental transit phasors. It should be clear, however, that just a single data trace would suffice to determine that the experiment matches much more closely with the quantum theory than the semiclassical theory. We plan to elaborate this result in separate publication [54].

\section{CONCLUSIONS AND FUTURE GOALS}

In summary, we have described the details of our recent experimental work to perform continuous measurement of the interaction energy $E_{\text {int }} \equiv \hbar g$ between one atom and an optical cavity during individual transit events. We displayed heterodyne transit signals in two complementary formats, one of which highlights the large bandwidth and nearly quantum-noise limited signal-to-noise ratio achieved in tracking the time-evolution of the amplitude and phase quadratures $\left(\tilde{x}_{a}, \tilde{x}_{p}\right)$ of light transmitted through the cavity. The second data format (transit phasors) displays the correlation induced between $\tilde{x}_{a}$ and $\tilde{x}_{p}$ by the atom-cavity interaction, and our experimental results show that we are able to distinguish unambiguously between quantum and semiclassical models of cavity QED in only a few (or even just one) atomic transits. We have furthermore presented the results of rudimentary numerical simulations of atomic motion under the influence of mechanical forces and momentum diffusion associated with the strong atom-cavity coupling, and we examined the interpretations suggested by these simulations for certain qualitative features of our experimental data (i.e. steps and asymmetries).

The primary conclusion we wish to draw in comparing the simulation results with our experimental data is that we have experimentally reached a regime of measurement sensitivity and bandwidth in which details of the atomic center-of-mass trajectories really should be visible. Even though we have no incontrovertible means of proving that steps and asymmetries seen in our experimental data should be associated with dynamical processes like channeling and diffusive escape, we are now motivated in our continuing work to develop some means of actively influencing the atomic motion while it is still inside the cavity. This would allow us to produce deliberate displacements of an atom along the cavity standing wave, and to examine the induced variations of the heterodyne signal in order to verify our inferred displacement sensitivity of $1.5 \times 10^{-10} \mathrm{~m} / \sqrt{\mathrm{Hz}}$. We anticipate that the combined abilities of monitoring and influencing atomic position relative to the cavity standing wave will enable the investigation of schemes for real-time feedback control of quantized atomic center-of-mass motion.

Finally, let us note that the standard quantum limit for overall observation time of the position of a single atom with sensitivity $1.5 \times 10^{-10} \mathrm{~m} / \sqrt{\mathrm{Hz}}$ and $300 \mathrm{kHz}$ bandwidth should be $t_{*} \sim 10 \mu \mathrm{s}$ [55. This implies that quantitative experimental investigations of conditional 
quantum dynamics (as described in 555) should indeed become possible in our cavity QED system once we are able to reliably prepare individual atoms in well-defined initial states of motion. The ideal situation in this regard would be to trap and localize atoms within the cavity, releasing them at a node or antinode of the standing wave, on the cavity axis, and with an initial position uncertainty that is small compared to $\lambda / 4$. Current efforts in our group focus on trying to achieve this level of control via optical dipole-force traps and/or far-detuned optical lattices inside the cavity.

We wish to acknowledge Q. A. Turchette's vital participation in the early stages of this work, and to thank A. C. Doherty, C. J. Hood, and T. W. Lynn for valuable discussions. Much of the numerical work described in this paper was performed on resources located at the Advanced Computing Laboratory of the Los Alamos National Laboratory. This research was supported by the National Science Foundation under Grant No. PHY9722674 , by the ONR, and by DARPA through the QUIC initiative (administered by ARO). Jun Ye is supported by an R. A. Millikan Fellowship from the California Institute of Technology.

[1] H. J. Kimble: in Cavity Quantum Electrodynamics. San Deigo, Academic Press: 1994

[2] D. M. Meekhof, C. Monroe, B. E. King, W. M. Itano, D. J. Wineland: Phys. Rev. Lett. 76, 1796 (1996)

[3] D. Leibfried, D. M. Meekhof, B. E. King, C. Monroe, W. M. Itano, D. J. Wineland: Phys. Rev. Lett. 77, 4281 (1996)

[4] W. E. Moerner: Science 265, 46 (1994)

[5] B. C. Stipe, M. A. Rezaei, W. Ho: Science 279, 1907 (1998)

[6] M. R. Andrews, D. M. Kurn, H. J. Miesner, D. S. Durfee, C. G. Townsend, S. Inouye, W. Ketterle: Phys. Rev. Lett. 79, 553 (1997)

[7] M. Kozuma, K. Nakagawa, W. Jhe, M. Ohtsu: Phys. Rev. A 57, R24 (1998)

[8] S. Guibal, C. Triche, L. Guidoni, P. Verkerk, G. Grynberg: Opt. Commun. 131, 61 (1996)

[9] C. M. Caves, G. J. Milburn: Phys. Rev. A 36, 5543 (1987)

[10] H. M. Wiseman: Quantum Semiclass. Opt. 8, 205 (1996)

[11] A. M. Herkommer, H. J. Carmichael, W. P. Schleich: Quantum Semiclass. Opt. 8, 189 (1996)

[12] A. C. Doherty, A. S. Parkins, S. M. Tan, D. F. Walls: Phys. Rev. A (in press)

[13] H. J. Carmichael, An Open Systems Approach to Quantum Optics. Berlin, Springer: 1993

[14] H. M. Wiseman, G. J. Milburn: Phys. Rev. A 47, 642 (1993)

[15] H. M. Wiseman, G. J. Milburn: Phys. Rev. A 47, 1652 (1993)
[16] P. Zoller, C. W. Gardiner: in Quantum fluctuations, Session LXIII of l'Ecole d'Ete de Physique des Houches. Amsterdam, North-Holland: 1997

[17] H. M. Wiseman: Phys. Rev. Lett. 75, 4587 (1995)

[18] R. Alicki, D. Makowiec, W. Miklaszewski: Phys. Rev. Lett. 77, 838 (1996)

[19] R. Schack, C. M. Caves: Phys. Rev. E 53, 3257 (1996)

[20] H. Mabuchi: Quantum Semiclass. Opt. 8, 1103 (1996)

[21] H. M. Wiseman: Phys. Rev. A 49, 2133 (1994)

[22] H. Mabuchi, P. Zoller: Phys. Rev. Lett. 76, 3108 (1996)

[23] J. A. Dunningham, H. M. Wiseman, D. F. Walls: Phys. Rev. A 55, 1398 (1997)

[24] K. S. Wong, M. J. Collett, D. F. Walls: Opt. Commun. 137, 269 (1997)

[25] A. H. Zewail: J. Phys. Chem. 100, 12701 (1996)

[26] S. D. Gensemer, P. L. Gould: Phys. Rev. Lett. 80, 936 (1998)

[27] M. A. M. Marte, P. Zoller: Appl. Phys. B 54, 477 (1992)

[28] P. Storey, T. Sleator, M. Collett, D. Walls: Phys. Rev. A 49, 2322 (1994)

[29] C. C. Gerry: Phys. Rev. A 53, 1179 (1996)

[30] H. Mabuchi, Q. A. Turchette, M. S. Chapman, H. J. Kimble: Opt. Lett. 21, 1393 (1996)

[31] C. J. Hood, M. S. Chapman, T. W. Lynn, H. J. Kimble: Phys. Rev. Lett. 80, 4157 (1998)

[32] M. J. Collett, R. Loudon, C. W. Gardiner: J. Mod. Opt. 34, 881 (1987)

[33] L. A. Lugiato: Prog. Opt. 21, 71 (1984)

[34] Superpolished BK7 substrates with multi-layer dielectric coatings fabricated by Research Electrooptics in Boulder, CO.

[35] A. E. Siegman: Lasers. Mill Valley, University Science Books: 1986.

[36] R. J. Rafac, C. E. Tanner, A. E. Livingston, K. W. Kukla, H. G. Berry, C. A. Kurtz: Phys. Rev. A 50, R1976 (1994)

[37] O. Schmidt, K.-M. Knaak, R. Wynands, D. Meschede: Appl. Phys. B 59, 167 (1994)

[38] Q. A. Turchette, R. J. Thompson H. J. Kimble: Appl. Phys. B 60, S1 (1995)

[39] J. H. Shirley: Opt. Lett. 7537 (1982)

[40] R. W. P. Drever, J. L. Hall, F. V. Kowalski, J. Hough, G. M. Ford, A. J. Munley, H. Ward: Appl. Phys. B 31, 97 (1983)

[41] W. Vassen, C. Zimmermann, R. Kallenbach, T. W. Hänsch: Opt. Commun. 75, 455 (1990)

[42] T. L. Boyd, H. J. Kimble: Opt. Lett. 16, 808 (1991)

[43] A. Cable, M. Prentiss, N. P. Bigelow: Opt. Lett. 15, 507 (1990)

[44] E. S. Polzik, J. Carri, H. J. Kimble: Appl. Phys. B 55, 279 (1992)

[45] G. Rempe, R. J. Thompson, H. J. Kimble: Opt. Lett. 17, 363 (1992)

[46] C. W. Gardiner: Quantum Noise. Berlin, Springer: (1991)

[47] S. Marksteiner, K. Ellinger, P. Zoller: Phys. Rev. A 53, 3409 (1996)

[48] A. C. Doherty: private communication (1996)

[49] H. Katori, S. Schlipf, H. Walther: Phys. Rev. Lett. 79, 2221 (1997)

[50] A. C. Doherty, A. S. Parkins, S. M. Tan, D. F. Walls: 
Phys. Rev. A 56, 833 (1997)

[51] Q. A. Turchette, C. J. Hood, W. F. Lange, H. Mabuchi, H. J. Kimble: Phys. Rev. Lett. 754710 (1995)

[52] M. Brune, F. Schmidt-Kaler, A. Maali, J. Dreyer, E. Hagley, J. M. Raimond, S. Haroche: Phys. Rev. Lett. 76, 1800 (1996)

[53] R. J. Thompson, Q. A. Turchette, O. Carnal, H. J. Kimble: Phys. Rev. A 57, 3084 (1998)

[54] H. Mabuchi, J. Ye, and H. J. Kimble: in preparation

[55] H. Mabuchi: Phys. Rev. A (in press)

FIG. 1. Schematic overview of the apparatus.

FIG. 2. Geometrical arrangement of the MOT beams relative to the physics cavity (figure not to scale). The mirror substrates are each $3 \mathrm{~mm}$ in diameter and $4 \mathrm{~mm}$ long. The MOT forms at a height of $\sim 7 \mathrm{~mm}$ above the cavity axis.

FIG. 3. A $15 \mathrm{~ms}$ segment of the typical recorded data, showing the simultaneously-recorded photocurrents proportional to the amplitude quadrature $\tilde{x}_{a}$ (upper trace) and phase quadrature $\tilde{x}_{p}$ (lower trace) of light transmitted through the physics cavity (see text). The experimental parameters for this data were $\Delta=10 \mathrm{MHz}, \Theta=0$, and $m=1.5$. The photocurrents were digitized at a rate of $10 \mathrm{MHz}$, with 12-bit resolution, and the analog bandwidth of the anti-aliasing filters was $300 \mathrm{kHz}$. Note the set of transient features clustered between 0.009 and 0.013 on the time axis, each of which was caused by the passage of an individual atom through the cavity.

FIG. 4. Cavity diffusion coefficients versus atomic position, for (from the top curve going down) atom-probe detuning $\Delta \equiv \nu_{a}-\nu_{p}=0,10,30$, and $50 \mathrm{MHz}$, with $m=2$ photons and $\Theta=0$.

FIG. 5. Cavity diffusion coefficients versus atomic position, for (from the top curve going down) $m=8,4$, and 2 photons, with the atom-probe detuning $\Delta=50 \mathrm{MHz}$ (again $\Theta=0$ ).

FIG. 6. (a) Simulated atomic trajectory and corresponding heterodyne signal for a single transit with $m=4, \Theta=0$, and $\Delta=50 \mathrm{MHz}$. Here $x$ is atomic position along the cavity standing-wave (measured in units of the optical wavelength) and $v_{x}$ is the atomic velocity along the standing-wave (measured in optical wavelengths per $\mu \mathrm{s}$ ). Note that the simulation includes three-dimensional classical center-of-mass motion for the atom, although only $x$ and $v_{x}$ are displayed above. The two subplots in part (b) display the expectation value of the intracavity phase quadrature amplitude $\left\langle q_{p}\right\rangle$ (which is proportional to $\tilde{x}_{p}$ ) filtered down to an analog bandwidth $\sim 300$ $\mathrm{kHz}$ (left), as well as $\left\langle q_{p}\right\rangle$ plus an appropriate amount of Gaussian noise to simulate shot noise in our heterodyne detection (right).
FIG. 7. Simulated atom-transit signals (see text), displayed at an analog bandwidth of $200 \mathrm{kHz}$. The atom-probe detuning $\Delta$ and probe power $m$ are indicated for each subplot, and the atom-cavity detuning $\Theta$ is zero in all cases. Upper traces represent the intracavity amplitude quadrature $\left\langle q_{a}\right\rangle$, with Gaussian noise added to reproduce the overall signal-to-noise ratio predicted by equation (10) (yielding a quantity proportional to $\tilde{x}_{a}$ ). Lower traces (with zero mean) represent the intracavity phase quadrature $\left\langle q_{p}\right\rangle$ plus Gaussian noise (proportional to $\tilde{x}_{p}$ ).

FIG. 8. Individual atom-transit signals, displayed at the full analog bandwidth of $300 \mathrm{kHz}$ and sampling rate of $10 \mathrm{MHz}$ (12 bit resolution). The atom-probe detuning $\Delta$ and probe power $m$ (see text) are indicated for each subplot, and the cavity-probe detuning $\Theta$ is zero in all cases. Upper traces represent the amplitude quadrature $\tilde{x}_{a}$, whereas the lower traces (with zero mean) represent the phase quadrature $\tilde{x}_{p}$. Note that we have displaced the $\tilde{x}_{a}$ traces by +400 in order to prevent them from overlapping with the $\tilde{x}_{p}$ traces, and that the photocurrents are displayed in arbitrary units.

FIG. 9. Dependence of transit phasor shapes on detuning (the values of probe power $m$ and detuning $\Delta$ are indicated above each subplot). Each subplot displays an overlay of two data traces (gray spots), quantum-mechanical theory from the Master Equation (solid curve ending in a o, computed using equation (1)), and semiclassical theory (computed using equation (7), solid curve ending in an " $\times$ ").

FIG. 10. Transit phasors for fixed detuning $\Delta=10 \mathrm{MHz}$, with variable probe strength (as indicated). Each subplot displays an overlay of three data traces (gray spots), quantum-mechanical theory from the Master Equation (solid curve ending in a $\circ$, computed using equation (1)), and semiclassical theory (computed using equation (7), solid curve ending in an " $\times$ "). 


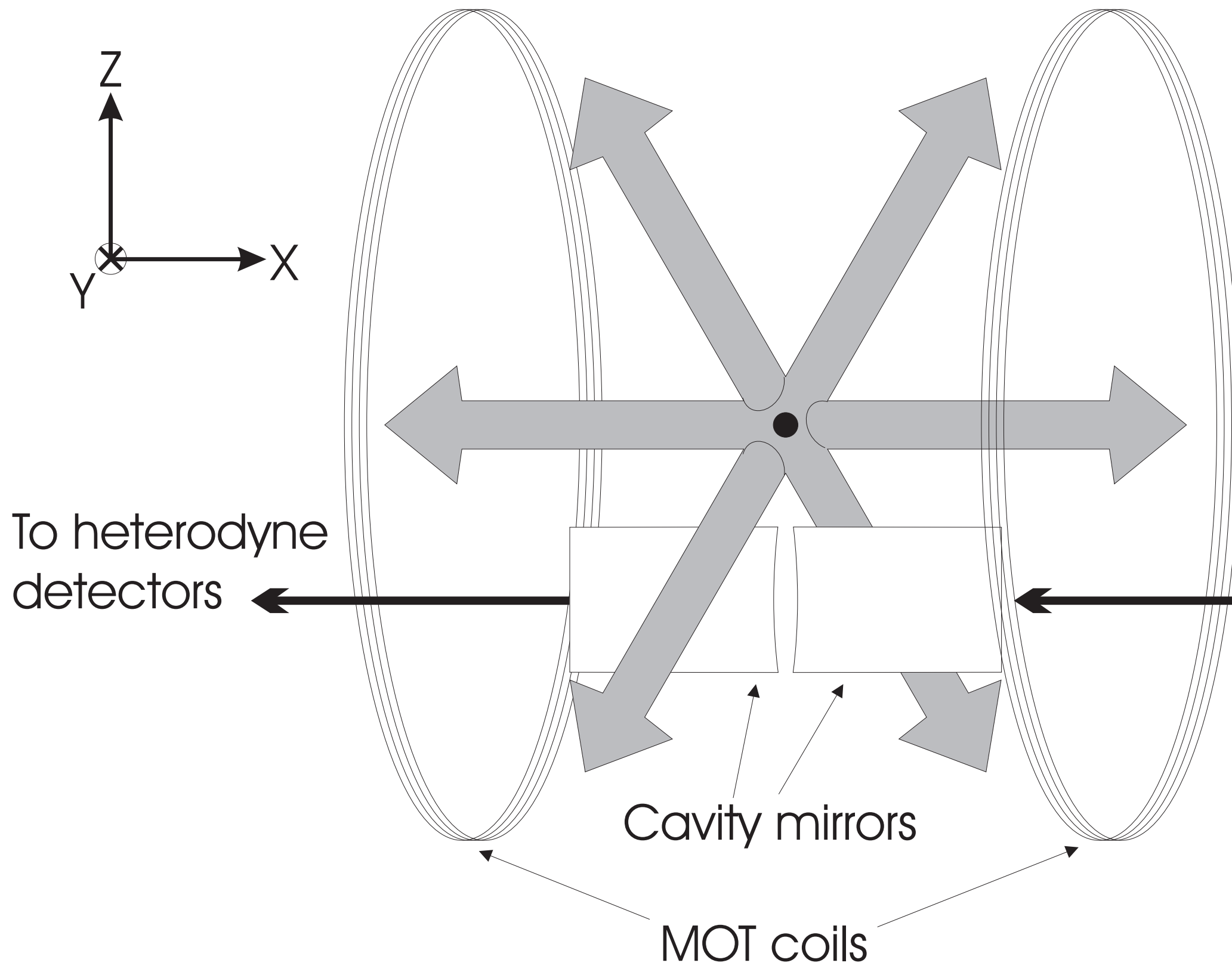




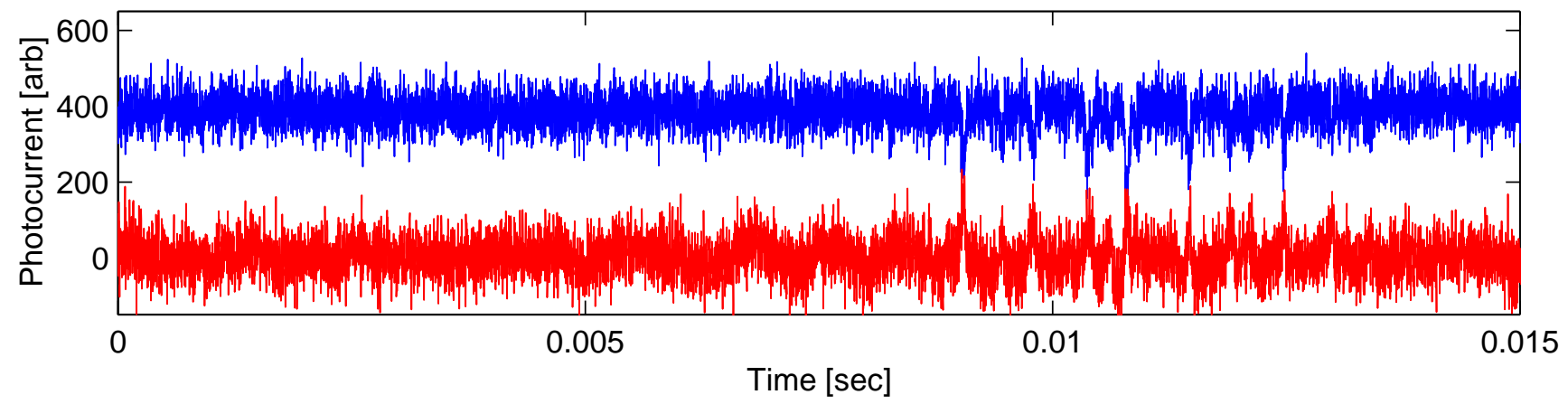




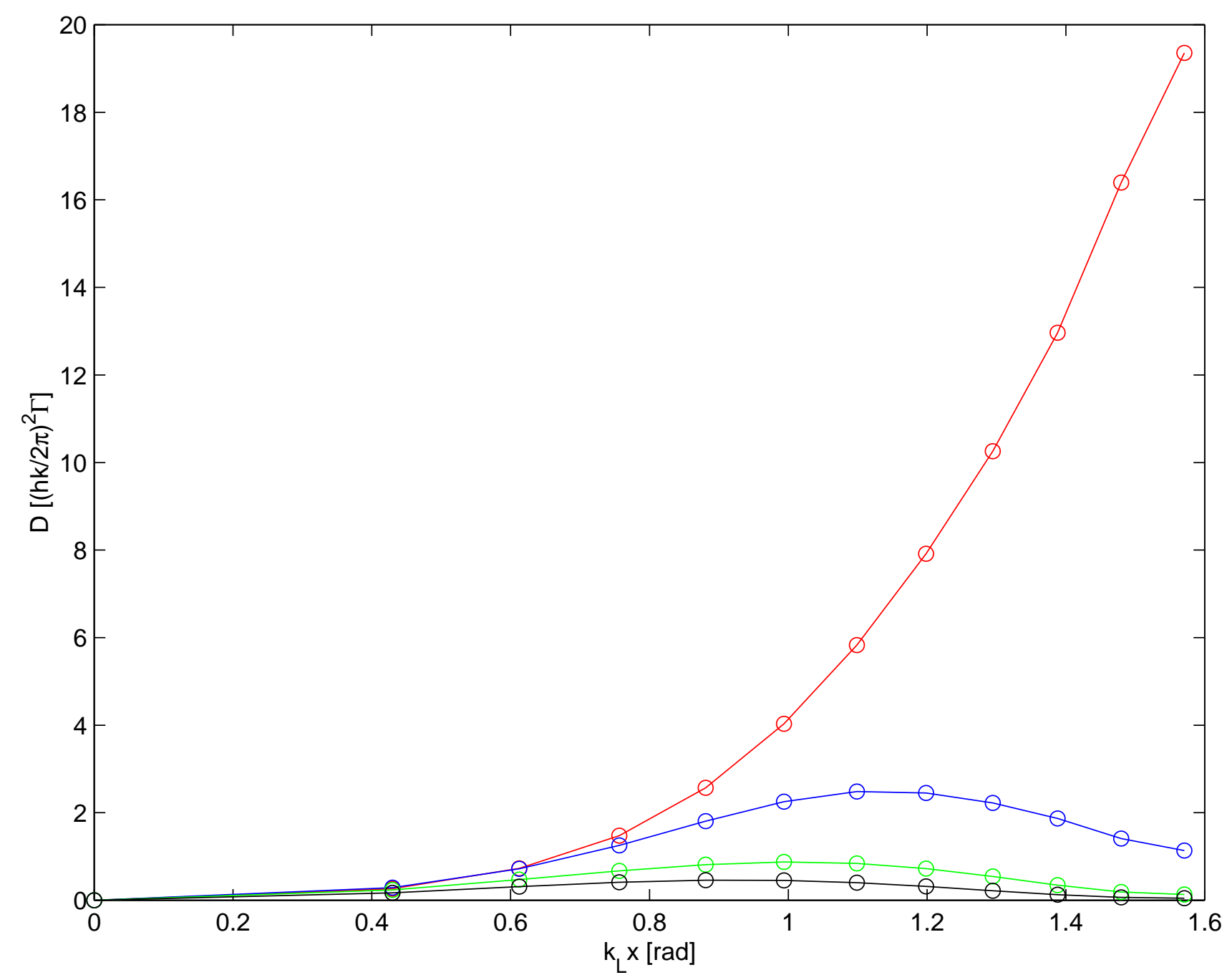




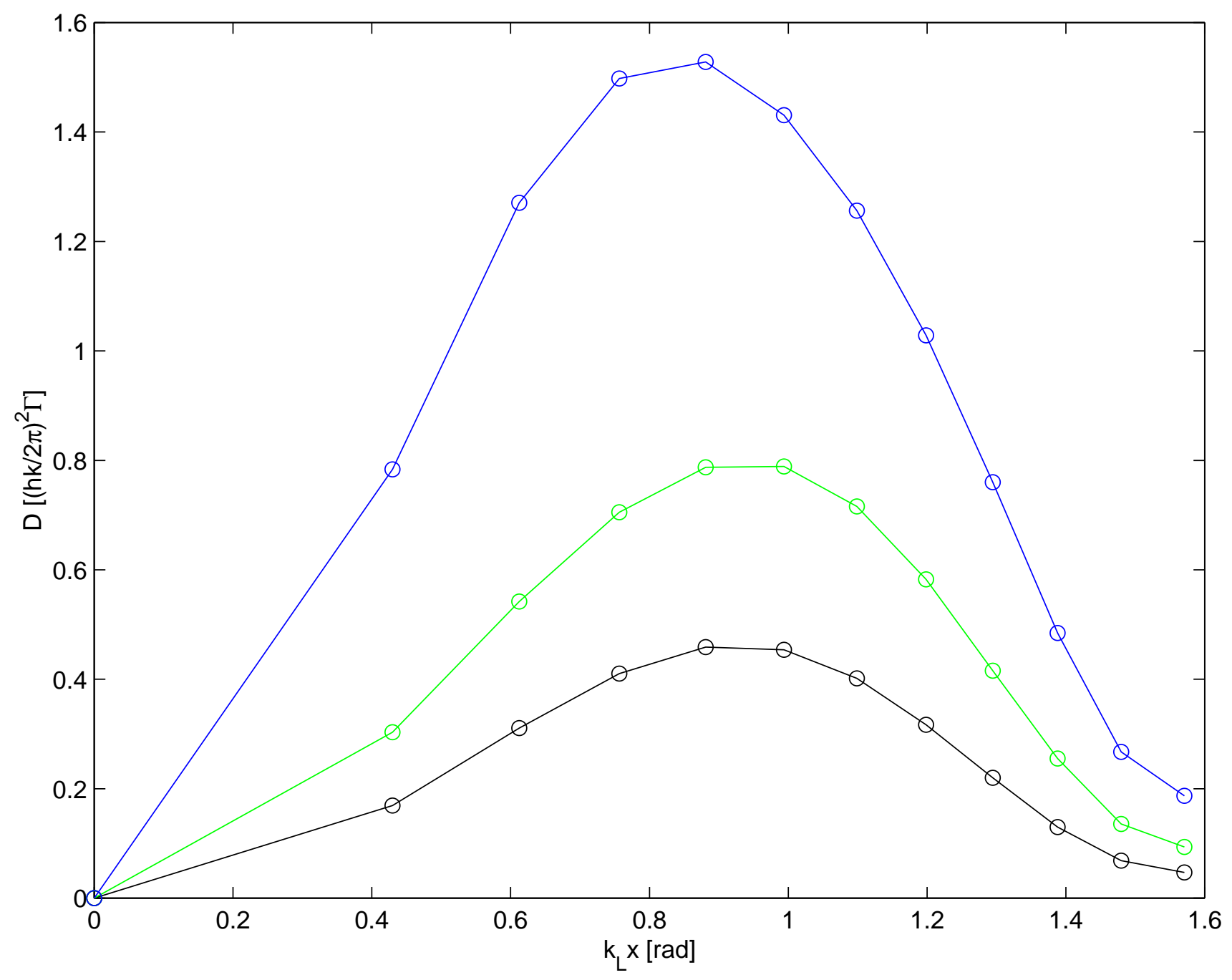



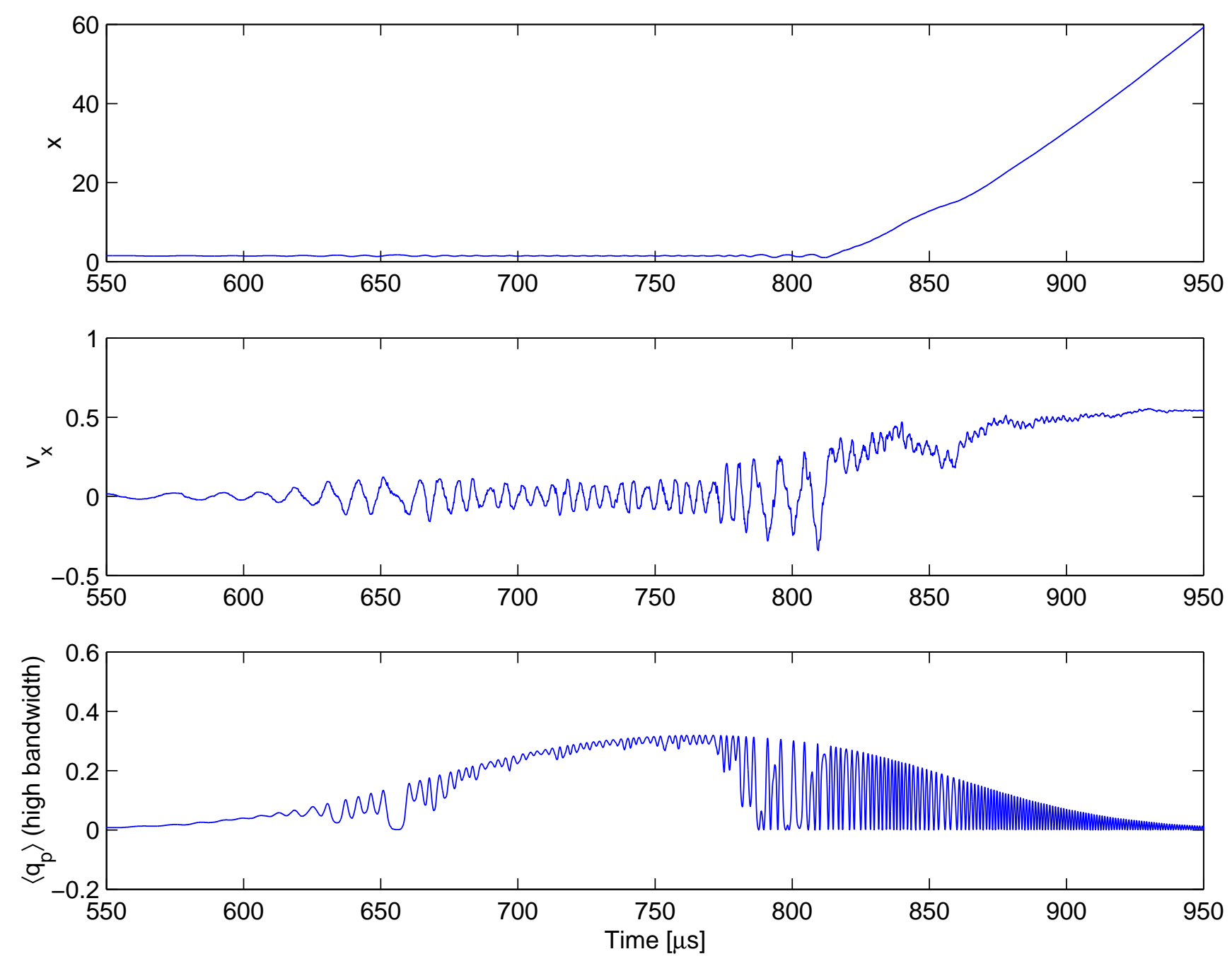

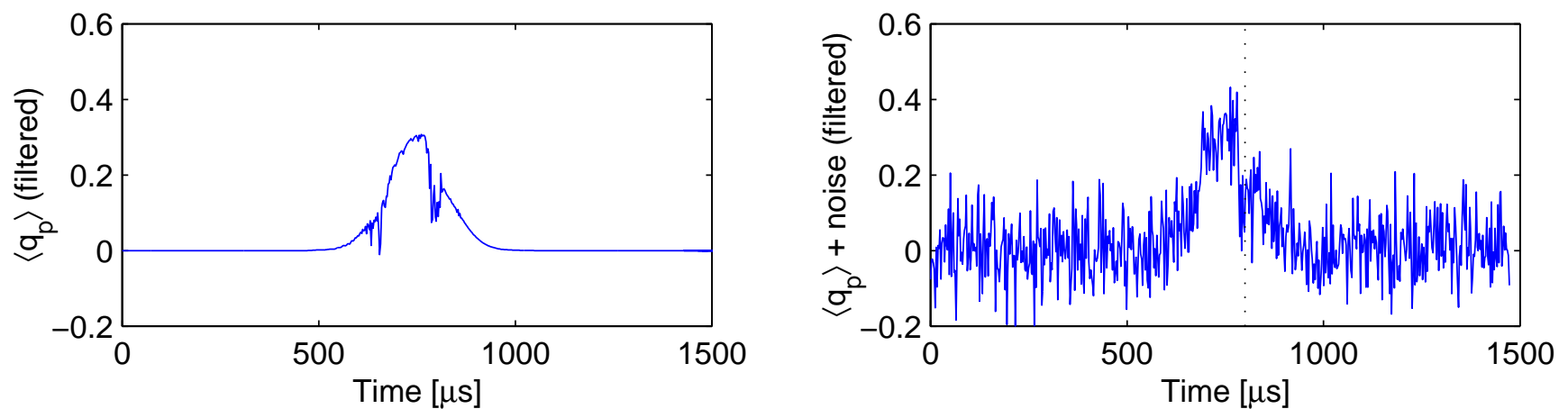

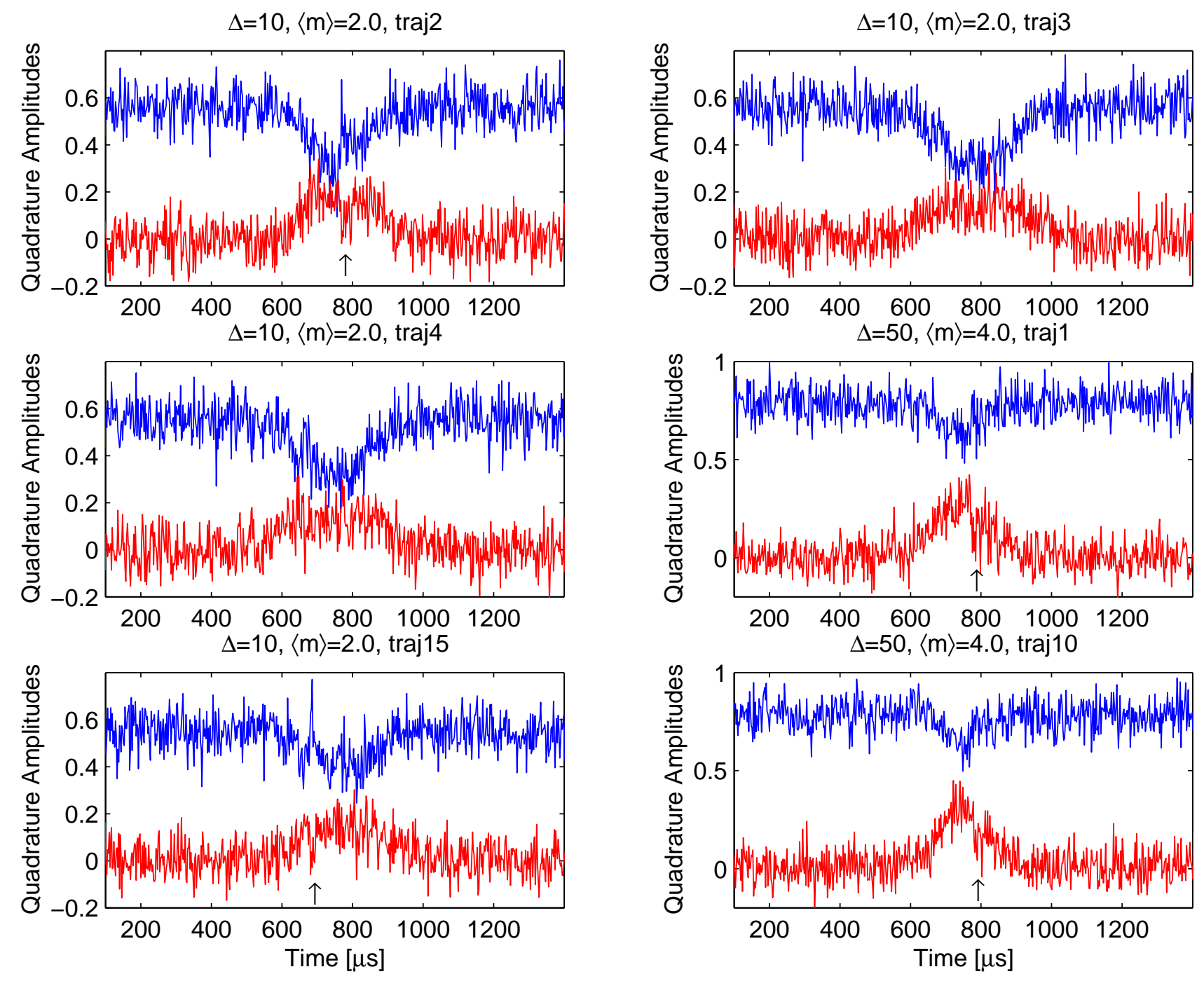

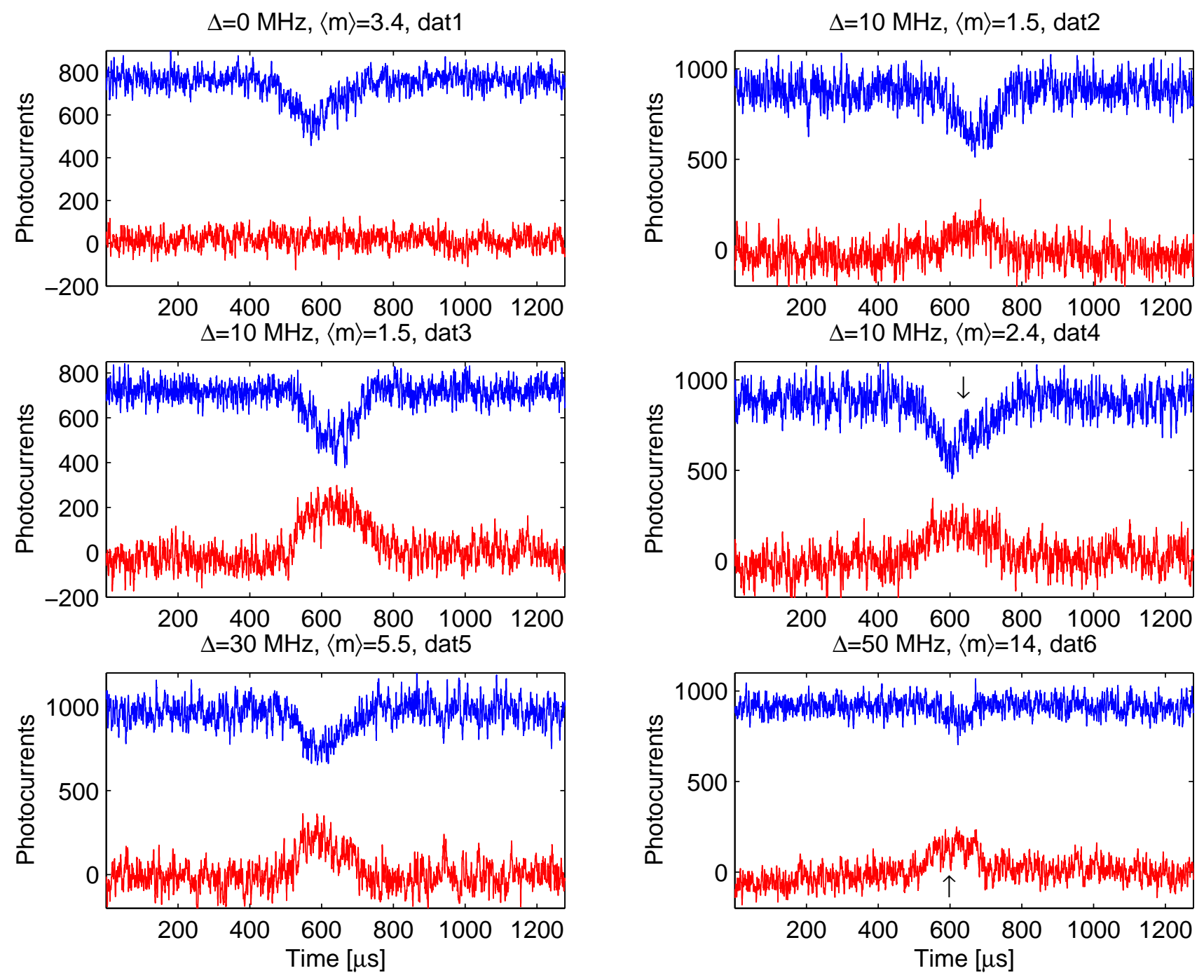

$\Delta=0$
$\Delta=10$
$\Delta=20$
$\Delta=30$
$m=3.4$
$m=2.8$
$m=4.4$
$m=5.5$
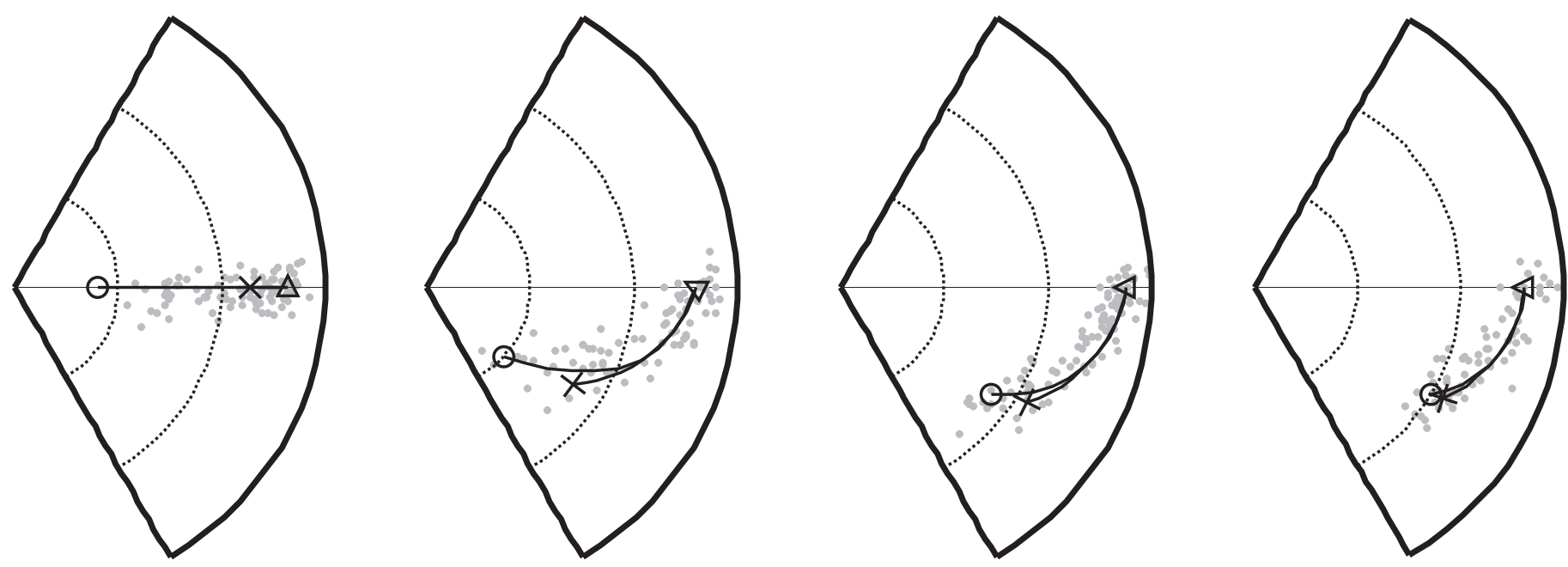

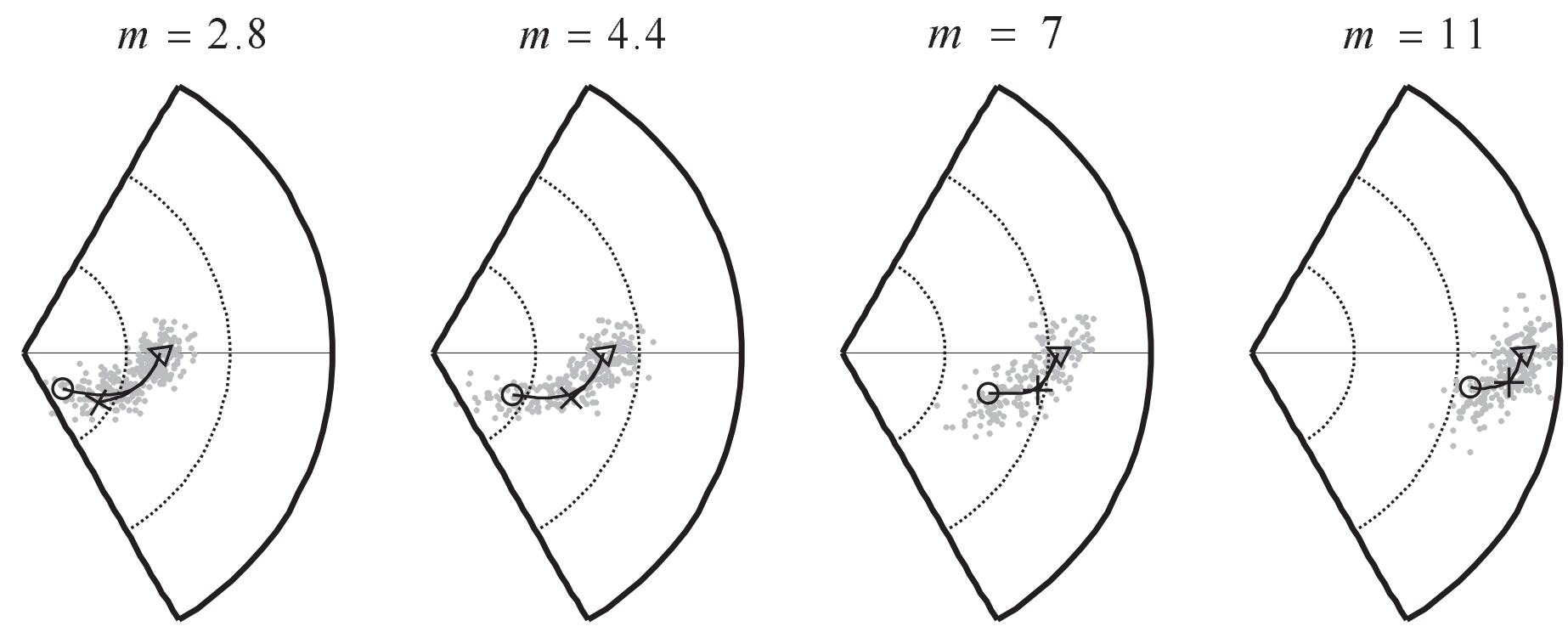